\title{
Jüngste Arbeiten zum Begriff der Dankbarkeit in Philosophie und Psychologie
}

\author{
Liz Gulliford · Blaire Morgan · Kristján Kristjánsson
}

Angenommen: 1. April 2021 / Online publiziert: 12. Mai 2021

(C) Der/die Autor(en) 2021

Zusammenfassung Der Beitrag gibt einen Überblick über die philosophische und psychologische Literatur zum Begriff der Dankbarkeit bis ins Jahr 2013. Geprüft werden die in beiden Wissenschaften veröffentlichten Arbeiten vor allem hinsichtlich ihrer begrifflichen Grundlagen und der ethischen Bewertung von Dankbarkeit, etwa als Pflicht, Tugend oder Supererogation. Die Analyse zeigt, dass jeweils mit einer Reihe untereinander unvereinbarer Begriffsverständnisse gearbeitet wird, sodass die Debatte von einem komplexen Netzwerk sich überschneidender und überkreuzender Begriffe geprägt ist. Der Beitrag endet mit Vorschlägen für die weitere Forschung. Psychologen wird empfohlen, ihre Konzeptionen von Dankbarkeit prä-

Der Aufsatz ist im Original unter dem Titel „Recent Work on the Concept of Gratitude in Philosophy and Psychology“ 2013 in The Journal of Value Inquiry 47, S. 285-317, erschienen. Autor und Autorinnen gehörten damals dem Jubilee Centre for Character and Values, School of Education, der University of Birmingham, UK, an. Die Literaturangaben zu damals noch im Erscheinen begriffenen Texten wurden hier aktualisiert.

Übersetzt von Veit Friemert. Anmerkung des Übersetzers: Einfache eckige Klammern, die englische Termini enthalten, z.B. [gratefulness], sind von mir und dienen der terminologischen Klärung. Eckige Klammern für Auslassungen, [...], oder mit deutschen Termini folgen dem englischen Original. Doppelte eckige Klammern, z.B. [[scheinbar]], sind von mir und betreffen Ergänzungen, die den Sinn der Argumentation hervorheben, aber so nicht im Original stehen. Runde Klammern für Auslassungen, (...), markieren Stellen, die in der Übersetzung durch die Herausgeber der ZEMO gestrichen wurden; dazu zählt u.a. der gesamte Abschnitt 9.

L. Gulliford $(\square)$

Centre for Psychology and Social Sciences, Faculty of Health, Education and Society, University of Northampton, University Drive, Northampton, NN1 5PH, Großbritannien

E-Mail: Liz.Gulliford@northampton.ac.uk

B. Morgan

School of Psychology, University of Worcester, Worcester, WR2 6AJ, Großbritannien

E-Mail: b.morgan@worc.ac.uk

K. Kristjánsson

Birmingham, Großbritannien 
ziser zu fassen und zu begründen sowie Bottom-up-Studien zum alltagspraktischen Begriff der Dankbarkeit durchzuführen. Philosophen sollten dagegen der in den Sozialwissenschaften durchgeführten Bottom-up-Arbeit mehr Beachtung schenken.

Schlüsselwörter Dankbarkeit · Emotion · Tugend · Pflicht · Supererogation · Triadischer vs. dyadischer Begriff

Dieser Aufsatz ist ein kritischer Bericht über die jüngste philosophische und psychologische Literatur zum Begriff der Dankbarkeit, die in den letzten Jahren eine Zunahme erfahren hat. In der Tat scheint es, dass sich gegenwärtig jeder für Dankbarkeit begeistern lässt. In theologischen Kreisen ist dies keine Neuigkeit. Seit der Mahnung des Paulus, ,,seid dankbar in allen Dingen; denn das ist der Wille Gottes“, haben die meisten in der christlichen Tradition arbeitenden Gelehrten der Dankbarkeit einen hohen Stellenwert zugemessen. Überraschender ist allerdings, dass in den säkularen Disziplinen der Psychologie und Philosophie ausgebildete Akademiker unlängst auf den Zug derjenigen aufgesprungen sind, die Dankbarkeit zu ihrem Thema gemacht haben. Die Zeiten sind vorbei, da Dankbarkeit als die „von Psychologen am meisten vernachlässigte Emotion“ galt und Philosophen zu Recht bemerkten, dass die gegenwärtige Philosophie ,relativ wenig über Dankbarkeit zu sagen" hat (Emmons 2004a, S. 3; McConnell 1993, S. vii). Plötzlich ist es zu einem beliebten Unterfangen in der Philosophie geworden, die begrifflichen Umrisse von Dankbarkeit zu eruieren, und Psychologen haben bereitwillig begonnen, die Beziehungen zwischen Dankbarkeit und einer Reihe positiver persönlicher und gesellschaftlicher Faktoren herauszuarbeiten. Viele dieser Psychologen entstammen dem Lager der jüngst etablierten Positiven Psychologie. Diese hat die Aufmerksamkeit innerhalb der Sozialwissenschaften auf eine Reihe von Themen verlagert, die sich im Umfeld von Dankbarkeit befinden und zu wenig beachtet worden waren, wie etwa Vergebung, Hoffnung und Optimismus, aber auch auf Begriffe, die für empirische Wissenschaftler heiße Eisen sind und zuvor vom Fach verbannt worden waren, wie der des moralischen Charakters und der Tugend (Allport 1937).

Was ist der Auslöser für diesen akademischen Orientierungswandel hinsichtlich der Dankbarkeit? Es scheint, dass die Zeit verschiedener Gründe wegen reif für deren Wiederentdeckung und Neubewertung war. Die zunehmende Aufmerksamkeit für die Tugendethik in der Moralphilosophie gegen Ende des zwanzigsten Jahrhunderts führte zu einer erneuten Untersuchung emotionaler Tugenden, und so war es nur eine Frage der Zeit, dass in diesem Zusammenhang Dankbarkeit Gegenstand der Aufmerksamkeit wurde. Gleiches gilt für die Psychologie. Hier waren die kognitive Revolution, die den Nachdruck auf komplexe kognitive Emotionen (im Unterschied zu bloßen Gefühlen) legt, verbunden mit der Schwerpunktsetzung auf der emotionalen Intelligenz zum Ende des zwanzigsten Jahrhunderts und auf den positiven Charakterzügen und Tugenden zu Beginn des einundzwanzigsten Jahrhunderts von Anbeginn dazu bestimmt, Dankbarkeit als einen Gegenstand möglichen Interesses zu ermitteln.

Was genau ist dann Dankbarkeit? Wie und wann zeigt sie sich? In welchem Sinne und warum sollte sie eine Tugend oder sozial nutzbringend sein? Diese Fragen 
beschäftigen gegenwärtig Philosophen ebenso wie Psychologen und keiner unter ihnen scheint eine einfache oder unproblematische Antwort zuzulassen. Einerseits ist es - obwohl die Tugendethik, insbesondere die aristotelische und neoaristotelische Tugendethik, momentan wirklich angesagt ist - in keiner Weise klar, wie Dankbarkeit allgemein in den Tugendrahmen passt, ganz zu schweigen in den Rahmen der aristotelischen Tugend (Curzer 2012). Sie erscheint weder im Katalog der Standardtugenden aus Aristoteles' Nikomachischer Ethik (Aristoteles 1999) noch unter den zusätzlichen Tugenden rein emotionaler Natur, die Aristoteles in seiner Rhetorik einführt (Aristoteles 2002). Darüber hinaus scheint er in seiner Beschreibung der Hochsinnigen (megalopsychoi) aus der Nikomachischen Ethik - der Vorbilder moralischer Tugend, denen wir offenkundig nacheifern sollen - die Dankbarkeit als eine mögliche Tugend zu verwerfen (NE IV 7-9 (1123a-1125a)). Selbst in dem Falle, dass die Dankbarkeit in einer ihrer vielen Bedeutungen in den Geltungsbereich einer aristotelischen Tugend gebracht werden könnte, würde dies wahrscheinlich eine erhebliche Reglementierung des Begriffs sowohl in Hinblick auf seinen akademischen wie alltagssprachlichen Gebrauch erforderlich machen. In der Tat hat einer der gegenwärtigen Autoren die Arbeit an diesem Projekt aufgenommen (Nisters 2012; Kristjánsson 2015).

Die Rede von verschiedenen Bedeutungen von Dankbarkeit trifft genau das, worum es hier geht und bringt uns direkt zum Thema der vorliegenden Abhandlung, nämlich jüngste Arbeiten zur Dankbarkeit in Hinblick auf deren begriffliche Grundlagen zu prüfen und Bedenken über den Zustand des Diskurses über Dankbarkeit zu äußern, die gravierender sind als dessen Abweichung vom Orakelspruch des Aristoteles. Wir kommen aus den Fachbereichen der Philosophie, Psychologie und Theologie und begannen unsere kritische Betrachtung der Literatur zur Dankbarkeit ausgehend von der Arbeitshypothese, dass der Begriff auf verschiedenen akademischen Feldern möglicherweise verschieden charakterisiert und untersucht wird was die interdisziplinäre Arbeit zur Dankbarkeit beschwerlich machen würde. Dies scheint, wie in Abschnitt 1 kurz dargestellt, offensichtlich der Fall zu sein. Darüber hinaus brachten unsere Nachforschungen allmählich eine größere Anzahl abweichender Verwendungsweisen des Dankbarkeitsbegriffs zum Vorschein, als wir uns ursprünglich vorgestellt hatten. Auch führten sie zu dem Befund, dass die Gelehrten im Bereich der Philosophie einerseits und in dem der Psychologie andererseits untereinander so gravierend unterschiedliche Meinungen vertreten und so deutlich aneinander vorbeireden, wie sie dies über ihre akademischen Grenzen hinaus tun. In der Tat scheinen sich die Begriffskontroversen über Dankbarkeit, die in der Philosophie wüten, mehr oder weniger in der Psychologie zu wiederholen, obwohl in weniger deutlich argumentativer Gestalt. Wir widmen uns diesen Kontroversen in den Abschnitten 2-8. Obwohl bereits von früheren Autoren die Meinungsverschiedenheiten beklagt wurden, die die fraglichen Diskursfelder kennzeichnen, und sie bestimmte begriffliche Defizite erkannt hatten, wird in Abschnitt 9 grundsätzlicher gezeigt, dass die Meinungsverschiedenheiten nicht allein verschiedene Auffassungen eines einzelnen Begriffs betreffen, sondern sich auf grundlegend verschiedene Begriffstypen beziehen (McAleer 2012; Lambert et al. 2009).

Die Schuld der Philosophen scheint darin zu bestehen, dass einige die von ihnen bevorzugten Annahmen zur Dankbarkeit im Namen begrifflicher Präzision oktroy- 
ieren und damit angeblich abweichende und deplatzierte Gebrauchsweisen spurlos beseitigen - ohne die Möglichkeit in Betracht zu ziehen, dass verschiedene Begriffe von Dankbarkeit im akademischen und Alltagsgebrauch vorkommen, deren jeder seine jeweils spezielle Funktion hat. Im Gegenzug scheint die Schuld der Psychologen darin zu bestehen, dass einige von ihnen ihre Arbeit entweder in einem begriffstheoretischen Vakuum verrichten oder eine spezielle Ausprägung von Dankbarkeit, die sie oft von einer respektierten Autorität übernehmen, als selbstverständlich voraussetzen, ohne sich die Mühe zu machen, Gründe für sie vorzubringen. Allein dadurch, dass man die verschiedenen Gebrauchsweisen des Terminus „Dankbarkeit“ sowie die Begriffskontroversen, die ihn umgeben, entwirrt, werden uns Fragen nach der Natur und Prominenz von Dankbarkeit zugänglich. Das Ziel der vorliegenden Abhandlung besteht somit nicht darin, eine die Literatur entlarvende Diagnose zu liefern, sondern darin, den psychologischen und philosophischen Darstellungen von Dankbarkeit einen Weg nach vorn aufzuzeigen, der aus den gegenwärtigen Sackgassen herausführt.

\section{Dankbarkeit über verschiedene Fachdisziplinen hinweg: divergierende Methoden und Interessen}

Ehe wir Auffassungen von Dankbarkeit untersuchen, die durch verschiedene Gelehrte vorgestellt und von ihnen als gegeben angenommen worden sind, ist es hilfreich, mit einem Überblick über die verschiedenen Schwerpunkte und Methoden zu beginnen, die jeweils von Philosophen und Psychologen gesetzt bzw. verwendet werden. Das Interesse der Philosophen am Begriff der Dankbarkeit hatte Fred Berger wiedererweckt. Philosophen nach Berger wenden üblicherweise die Methode der Begriffsanalyse bei ihrer Analyse von Dankbarkeit an, die sich bereits in ihrem Werkzeugbestand befindet, und behandeln den Gegenstand auf dieselbe Weise, wie sie andere strittige Begriffe in Moral- und Sozialphilosophie behandeln (Berger 1975). Die übliche Hintergrundannahme besteht darin, dass die Begriffsanalyse (der passenden philosophischen Art) eine Methode ist, die sich in hohem Maße dafür eignet, die Struktur der menschlichen Erfahrungen in einer gemeinsam geteilten natürlichen und soziomoralischen Welt zu enthüllen (Kristjánsson 1996, Kap. 7; siehe auch Roberts 2003, Kap. 1.5). Die für die Anwendung dieser Methode bedeutendsten Publikationen sind Terrence McConnells Buch aus dem Jahre 1993 - aufgrund seiner auschlaggebenden Reichhaltigkeit, seines breiten Anwendungsbereichs und der Fülle seiner Szenarien, die die Intuition schulen und Einsichten vermitteln - und Robert C. Roberts' prägnante und präzise argumentierende Abhandlung aus dem Jahre 2004 (McConnell 1993; siehe auch Roberts 2004). Als einschlägigen Fall betrachte man folgende strikte Charakterisierung, die Roberts nach der Vorstellung dessen geliefert hat, was er ,begriffliche Stützung“ (im Unterschied zu ,empirischer Stützung“) für einen jeden einzelnen Aspekt des Ausdrucks „Ich bin S für X dankbar“" nennt (Roberts 2004, S. 59-64): 
1. $\mathrm{X}$ ist für mich von Nutzen ( $\mathrm{X}$ zu haben ist mir wichtig).

2. $S$ hat gut gehandelt, indem er mir $X$ gegeben hat (es ist wichtig für mich, dass $S$ mir X gegeben hat).

3. Indem $\mathrm{S}$ mir $\mathrm{X}$ gegeben hat, ist er über das hinausgegangen, was er mir schuldet und setzt mich regelrecht in seine Schuld (ich bin bereitwillig in der Schuld von S).

4. S hat mir gegenüber wohlwollend gehandelt, indem er mir $\mathrm{X}$ gegeben hat $(\mathrm{S}$ ' Wohlwollen, das darin zum Ausdruck kommt, dass er mir X gegeben hat, ist mir wichtig).

5. Dass $S$ mir gegenüber wohlwollend ist und mir $X$ gegeben hat zeigt, dass er gut ist (ich fühle mich zu S hingezogen). [Oder: Die Freundlichkeit von $\mathrm{S}$ zeigt, dass $\mathrm{X}$ gut ist und dass $\mathrm{S}$, indem er mir $\mathrm{X}$ gibt, mir gegenüber wohlwollend ist.]

6. Ich möchte meiner Verbindlichkeit und Zuneigung $S$ gegenüber durch eine symbolische Gegenleistung Ausdruck verleihen.

Mit begrifflicher Stützung meinen Philosophen üblicherweise Erwägungen, die sich aus der Erkundung der Intuitionen (linguistisch) kompetenter und (philosophisch) reflektierter Akteure hinsichtlich der Frage ergeben, wie ein gegebener Begriff möglichst nutzbringend verwendet wird - um Begriffsverwirrungen zu minimieren - und wie sich dieser Begriff in möglichst vernünftiger Weise von angrenzenden Begriffen auf dem linguistischen Terrain unterscheiden lässt. Die Beiträge von McConnell und Roberts verkörpern diese von den meisten Philosophen bevorzugte Untersuchungsmethode. Sie zeigen aber auch auf, wie Philosophen dazu neigen, Dankbarkeit primär als einen moralischen Begriff zu betrachten und ihre Aufmerksamkeit darauf zu richten, die moralische Vorzugswürdigkeit und Rechtfertigung dieses Begriffs aufzuklären sowie darauf, welche Folgerungen aus ihm zu ziehen sind.

Angesichts des philosophischen Strebens nach begrifflicher Strenge lässt sich fragen, warum Untersuchungen des Begriffs der Dankbarkeit zu so vielen und voneinander abweichenden Auffassungen des Begriffs geführt haben, die in den Abschnitten 2-8 aufgezeigt werden. Naheliegend sind die folgenden Möglichkeiten, die sich entweder aus der Art der verwendeten Methode oder ihrer Anwendung in diesem bestimmten Fall erklären. Erstens hat Carr vermutet, ob Philosophen nicht typischerweise dem essentialistischen Streben nach einer Einheitsdefinition eines hoffnungslos proteischen Terminus zum Opfer fallen, was sich im dogmatischen Beharren darauf zeigt, alle Widersprüche unter Kontrolle zu bringen und aufzulösen (Carr 2013). Zweitens lassen sich womöglich die Intuitionen kompetenter und reflektierter Sprecher in Hinblick auf Dankbarkeit letztlich nicht systematisieren und unter einen Hut bringen. Drittens ist vielleicht an der Art und Weise, wie die philosophische Begriffsanalyse normalerweise sogenannte Basisintuitionen untersucht, generell etwas nicht in Ordnung. Um die wirkliche Grammatik moralischer Termini zu ergründen, werden uns entweder schematische Gedankenspiele geboten, die oft in nur wenigen Sätzen imaginäre Personen in einer bestimmten moralisch aufgeladenen Situation zeigen, woraufhin wir dann zu einer angemessenen Beurteilung der Situation aufgefordert werden. Oder man verweist uns an Charaktere aus wohlbekannten Romanen, was uns wohl eher unmittelbarere, vertrautere und leben- 
digere Darstellungen der Situation und ihrer Bildungselemente verschaffen soll. McConnell hat den ersteren Weg zu den Intuitionen beschritten, Roberts den letzteren (McConnell 1993). ${ }^{1}$ Beide Verfahrensweisen haben allerdings ihre Probleme. Schematische Gedankenspiele sind oftmals zu abstrakt, um unzweideutig die Merkmale der moralischen Grammatik herauszustellen, nach denen man sucht. Dies gilt wohl für viele der von McConnell skizzenhaft gezeichneten Beispiele und Fragen danach, ob in ihnen Fälle von Dankbarkeit vorliegen oder nicht. Zweitens muss ungeachtet der Tatsache, dass große Literatur generell das Leben reflektiert und grundlegende Einblicke in das Seelenleben wirklicher Menschen eröffnen kann, in Betracht gezogen werden, dass Schriftsteller oftmals Überspitzungen als Mittel der Darstellung nutzen. Im Ergebnis kann dann etwas entstehen, das eher eine Karikatur als einen Einblick in die Charaktere wirklicher Menschen darstellt. Roberts Untersuchung von Dickens Bleak House ist hier vielleicht ein typisches Beispiel. Die dort erkennbaren miteinander verschränkten Pathologien von Dankbarkeit und Großzügigkeit teilen uns möglicherweise in idealisierter Weise etwas über die Umrisse dieser verwandten Tugenden mit, was allerdings vielleicht nicht die Weise widerspiegelt, in der man ihnen üblicherweise im Alltag begegnet (Roberts 2012, 2013).

Viele Sozialwissenschaftler werden umgehend darauf verweisen, dass für diese potentiellen Fallstricke der Begriffsanalyse dieselben weltfremden und paternalistischen Einstellungen von Philosophen verantwortlich sind, die die begriffliche Strenge und Sparsamkeit gegenüber der Reichweite vorziehen und die nur selten Belegmaterial dafür berücksichtigen, wie Begriffe tatsächlich alltagssprachlich verwendet werden. Dieser Kritik zufolge laufen sogenannte terminologische Reglementierungen, wie sie von Philosophen praktiziert werden, Gefahr, den Alltagsgebrauch der Sprache so weit zu verengen, dass sie austrocknet. Uns blieben dann nichts als obskure, abgedroschene und letztlich irrelevante Definitionen, die ihrer analytischen Schärfe zum Trotz kaum mehr als philosophische Spielereien wären (Gregg et al. 2008). Obwohl wir zögern, solchen harschen Kritiken der Begriffsanalyse als einer philosophischen Methode beizupflichten, so funktioniert diese Methode doch am besten, wenn sie auf einen bestimmten Begriffstyp Anwendung findet - auf naturalistische Begriffe mit einem eindeutigen Kern, aber unscharfen und strittigen Grenzen. Aber Dankbarkeit dem alltäglichen Verständnis nach ist ein solcher Begriff nicht. Wir werden uns dieser Thematik in Abschnitt 9 erneut zuwenden, nachdem wir die entsprechenden linguistischen und theoretischen Belege erörtert haben.

Psychologen bringen andere und in der Tat vielfältigere Interessen und Methoden als Philosophen in die Diskussion ein. Statt sich mit Fragen begrifflicher Klarheit und moralischer Rechtfertigung zu beschäftigen, sind Psychologen in Fragen der Dankbarkeit ganz auf das Erfordernis konzentriert, Dankbarkeit empirisch zu quantifizieren und zu messen, um deren Beziehung zu anderen Variablen aufzuklären, insbesondere zum subjektiven Wohlbefinden. Dieser Schwerpunkt darauf, Auswirkungen der Dankbarkeit offenzulegen, hat die Verwendung abhängiger Variablen erforderlich gemacht, insbesondre jener, die mit körperlicher und geistiger Gesundheit in Beziehung stehen. Damit soll innerhalb des vorherrschenden und kulturell

\footnotetext{
1 Siehe auch Roberts 2012 und „Generosity and Gratitude in Bleak House“, Vortrag auf dem Jubilee Centre
} Seminar, University of Birmingham, 6. Februar 2013. 
geschätzten Paradigmas empirischer Wissenschaft gezeigt werden, dass Dankbarkeit einen erkenn- und messbaren Einfluss auf menschliches Tun hat. So behauptet Robert Emmons, nur eine „,wissenschaftliche Perspektive“ könne einen „evidenzbasierten Zugang" zu den Entsprechungen von Dankbarkeit liefern (Emmons 2012, S. 12). Zwei Instrumente beherrschen das Feld, beides Selbsteinschätzungsfragebögen, in denen Menschen Aussagen wie etwa „Ich bin vielen verschiedenen Menschen dankbar" anhand der Lickert-Skala bewerten: Die eine nennt sich GratitudeQuestionnaire-6 oder GQ-6, die andere Gratitude, Resentment und Appreciation Test oder GRAT (McCullough/Emmons/Tsang 2002; siehe auch Watkins et al. 2003). (...)

Abgesehen von der schon genannten Betonung auf der Quantifizierung neigen Psychologen dazu, Dankbarkeit als eine Quelle positiver (angenehmer) Erfahrungen zu betrachten und deren Beziehungen mit anderen positiven (angenehmen und/ oder prosozialen) Variablen zu bewerten. Untersuchungen zu Wechselbeziehungen (zum Beispiel zu einer Verbindung von Dankbarkeit und subjektivem Wohlbefinden, im Folgenden SWB) gelingt es selten, Kausalbeziehungen festzustellen, etwa ob Dankbarkeit glücklich macht oder Glück dankbar werden lässt. Dieser Mangel betrifft allerdings weder die Dankbarkeitsstudien allein, noch beeinträchtigt er wesentlich deren Verdienste. Wir hatten erwartet, hier eine größere Sensibilität gegenüber den alltagspraktischen Vorstellungen von Dankbarkeit zu finden als in der philosophischen Literatur. In der Tat haben wir in einigen Bereichen der psychologischen Dankbarkeitsforschung eine größere begriffliche Offenheit als bei ihrem philosophischen Gegenüber festgestellt. So sind in der Tendenz Psychologen eher als Philosophen bereit, eine umfassende Charakterisierung von Dankbarkeit im Sinne eines dyadischen Begriffs statt eines engeren triadischen zu akzeptieren, was sich in den obengenannten Instrumenten widerspiegelt. Wir würden allerdings zögern, aus diesem Befund zu schließen, dass die fraglichen Psychologen in ihrer Forschung eine größere begriffliche Offenheit deshalb in Betracht ziehen, weil sie der Umgangssprache eine größere Achtung entgegenbringen. Tatsächlich zeichnet sich die sozialwissenschaftliche Literatur zur Dankbarkeit durch einen generellen Mangel an Aufmerksamkeit für Begriffsfragen aus. Mit Ausnahme einer Untersuchung, auf die wir später mehrfach zurückkommen werden, scheint im Bereich der Sozialwissenschaften nur sehr wenig Arbeit auf die Frage verwendet zu werden, was Menschen gemeinhin mit dem Terminus „Dankbarkeit“ meinen (Lambert, Graham und Fincham 2009). Bezeichnenderweise untersucht keines der Instrumente zur Messung der Dankbarkeit die Begriffsverständnisse der Probanden. Auch scheint bedauerlicherweise (wie wir zu gegebener Zeit zeigen werden) die Aussage nicht gerechtfertigt zu sein, dass die Konstrukteure jener Instrumente ,starke theoretische Gründe in Anschlag gebracht haben, um eine Apriori-Konzeption zu entwickeln“ (Wood, Maltby, Stewart, Joseph 2008a, S. 623).

Wozu hingegen die Angebote in der psychologischen Literatur neigen, ist eine der folgenden Alternativen oder eine Kombination derselben: (a) Die fraglichen Forscher haben das Gefühl und nehmen an, dass dankbaren Menschen bestimmte Eigenschaften zukommen (Watkins et al. 2003). (b) Definitionen werden von bekannten Dankbarkeitsforschern wie McCullough und Emmons übernommen (ohne hinreichend in Betracht zu ziehen, wie sich diese Definitionen erweitern oder verbessern ließen), die wiederum auf frühere Forscher und deren Begriffsverständ- 
nisse rekurrieren. Die Untersuchung von 26 jüngst erschienenen Abhandlungen zur Dankbarkeit, die von Psychologen verfasst worden sind, erbrachte, dass 10 die von Emmons und Mitarbeitern angebotene Definition zitieren und 12 Definitionen nutzen, die vordem von McCullough und Mitarbeitern vorgeschlagen worden waren. ${ }^{2}$ Bemerkenswerterweise findet sich in vier Abhandlungen keine Definition von Dankbarkeit, wobei diese Abhandlungen dann aber dazu übergehen, deren Korrelate oder Vorzüge zu untersuchen (Froh et al. 2011; Wood et al. 2007, 2008a, b, 2009). Nur in fünf Abhandlungen hatte man sich bemüht, eine eigene Definition von Dankbarkeit einzuführen (Seligman et al. 2005; Tsang 2006; Tsang et al. 2012; Wood et al. 2010). (c) Am meisten enttäuscht - obwohl häufig Lippenbekenntnisse hinsichtlich der Schwierigkeiten einer angemessenen Umschreibung nicht fehlen -, dass in der Literatur Phrasen wie „Dankbarkeit ist neulich als ... konzipiert worden“ oder ,es herrscht dahingehend Übereinstimmung, dass [Dankbarkeit X] ist“"im Überfluss vorgebracht werden, ehe die Forscher sich kopfüber in die Aufgabe stürzen, ein Instrument zu entwerfen oder eine Diskussion über empirische Erkenntnisse zu führen (Gordon, Musher-Eizenman, Holub, Dalrymple 2004).

Generell ist es problematisch, auf persönliche Intuitionen zu setzen, insbesondere aber hier, weil die fraglichen Psychologen üblicherweise darauf vertrauen, ihre Intuitionen zu äußern statt für sie Gründe vorzubringen. Darüber hinaus setzt sich die Bezugnahme auf jene, die in diesem Forschungsbereich einen großen Namen haben, der Gefahr eines Dominoeffekts aus, wenn sich nämlich erweisen sollte, dass der Theoretiker, bei dem dann die Verantwortung liegt, im Irrtum ist und der Verweis auf das, was in der früheren Forschung geläufige Annahmen waren, überkommenes Wissen verdinglicht. Während also Sozialwissenschaftler ihren Unmut über hochfliegendes, aprioristisches, durch empirische Beschränkungen unbehindertes Philosophieren äußern mögen, finden Philosophen in der psychologischen Forschungsliteratur zur Dankbarkeit möglicherweise nur wenig mehr als, um hier Wittgensteins harsche Kritik zu zitieren, „Begriffsverwirrung“ und den Verdacht, dass „die Sprache feiert" - was passiert, wenn man den empirischen Karren leichtfertig vor das Pferd spannt (Wittgenstein 1984, S. 260 und 580). Letztendlich gelangen wir somit auf zwei Diskursfelder zum Thema Dankbarkeit (in der Philosophie und der Psychologie), auf denen jeweils das Interesse dafür fehlt, was Menschen tatsächlich mit Dankbarkeit meinen. (...)

\section{Handlung, Emotion, Episode oder Charakterzug?}

Eine zumindest bis auf Thomas von Aquin zurückgehende Ansicht besagt, Dankbarkeit sei im Wesentlichen die Bezeichnung für eine bestimmte Handlung - nämlich die der Bezeugung von Dank. Gleichermaßen ist in den frühesten psychologischen Schriften zur Dankbarkeit keine Rede von einer erforderlichen emotionalen Antwort.

\footnotetext{
2 Zu den Abhandlungen, die auf Emmons 2004a bezugnehmen, gehören: Emmons 2004b; Emmons 2007; Emmons und Crumpler 2000; Emmons und McCullough 2003; Emmons und Shelton 2002; McCullough, Emmons, Tsang 2002; McCullough, Kilpatrick, Emmons, Larson 2001; McCullough, Kimeldorf, Cohen 2008; McCullough, Tsang, Emmons 2004.
} 
Bertocci und Millard zufolge ist Dankbarkeit „die Bereitschaft anzuerkennen, dass man der Begünstigte von jemandes Gefälligkeit ist, ob nun eine emotionale Antwort vorliegt oder nicht“ (Bertocci, Millard 1963). Mit dem Aufkommen kognitiver Emotionstheorien in der Psychologie und der Neubelebung der von Aristoteles inspirierten Tugendethik in der Moralphilosophie im späten zwanzigsten Jahrhundert wurden kognitionsbasierte Emotionen in Theorien der Humanpsychologie und des „guten Lebens“ integriert.

McConnells philosophische Untersuchung aus dem Jahre 1993 präsentiert uns diese Übergangsphase. Während bei ihm die Ansicht, dass gewisse öffentliche Bekundungen notwendig für Dankbarkeit sind, beibehalten wird, betont er auch die sogenannten innerlichen Aspekte: Die Erwiderung von Liebenswürdigkeiten und das öffentliche Zum-Ausdruck-Bringen von Dankbarkeit stellen die äußeren Verhaltensmerkmale von Dankbarkeit dar. Die Anerkennung, die Bestätigung und die Wertschätzung der Absichten desjenigen, der Dankbarkeit stiftet, bildet den innerlichen Aspekt: Gründe für und Gefühle der Dankbarkeit. Zur Dankbarkeit [gratitude] gehört folglich zwangsläufig, ein Gefühl der Dankbarkeit [gratefulness] vollständig und glaubwürdig zu verwirklichen. Es reicht nicht, einfach Dank zu bezeugen oder Geschenke zu erwidern (McConnell 1993, S. 56-61). Obwohl Dankbarkeit entwicklungsgeschichtlich in Austauschbeziehungen unter Primaten gründen mag, geht sie doch über einen bloßen Austausch gegenseitiger Vorteile hinaus. Dies unterstreicht, im Unterschied zur relativ unpersönlichen Natur der Wechselseitigkeit, die subjektive, persönliche und relationale Natur von Dankbarkeit.

Obwohl das Versagen, ethisch zu handeln, nach Bestrafung verlangen kann, seien, so macht McConnell weiter geltend, innerliches Versagen des Urteilens und des Fühlens die angemessenen Gegenstände des Vorwurfs. Während viele Ethiktheorien (nicht aber Tugendethiken) nur auf Handlungen setzen, schließt er, etwas würde ethisch gesehen fehlen, wenn ein Individuum einen Mangel an Gefühl in Bezug auf eine Episode feststellen sollte, in der man Dankbarkeit für angemessen hielte. Entsprechendes gilt für Vergebung: Sollte eine Person ohne korrespondierende Gefühle der Vergebung behaupten, sie hätte einer anderen vergeben, würden wir an der Glaubwürdigkeit der Vergebung zweifeln. Wir möchten allerdings hervorheben, dass hier zwischen den emotionalen, konativen und kognitiven Aspekten von Vergebung möglicherweise keine eindeutige Beziehung [a dislocation] besteht (Gulliford 2013).

Es ist nicht ganz klar, ob die Alltagssprache zwei Begriffe enthält: einen Begriff der Dankbarkeit als Handlung und einen der Dankbarkeit als Emotion. Eine Alternative könnte sein, dass man sich mit Äußerungen wie „P zeigte Dankbarkeit, obwohl P nicht wirklich dankbar war" auf denselben Begriff bezieht, wie man sich vermutlich auch auf denselben Begriff von Liebe bezieht, wenn man äußert, ,P bekundete Liebe, obwohl er nicht wirklich liebte“. Eine weitere Möglichkeit könnte sein, dass inkompetente Sprecher das Wort „Dankbarkeit“ zuweilen falsch verwenden, wenn sie stattdessen einen Terminus wie etwa „Erwiderung“ hätten nutzen sollen (wie wenn man so handelt, als ob man dankbar wäre, jedoch ohne die damit einhergehende Emotion). Eine weitere Möglichkeit bestände darin, dass es sich hier tatsächlich um zwei in der Alltagssprache weit verbreitete Begriffe handelt, einen der Handlungsdankbarkeit und einen der Emotionsdankbarkeit. Dies ließe sich dadurch 
ermitteln, dass man kompetente Sprecher mit Szenarien konfrontierte, in denen $\mathrm{P}$ den Erhalt einer Wohltat anerkennt und diese Wohltat entweder mit Widerwillen erwidert oder nur deswegen, um einen guten Eindruck zu hinterlassen, und sie fragte, ob diese Szenarien als Fälle von Dankbarkeit zählen. Dieser ungeklärte Sachverhalt liegt jedoch außerhalb unseres gegenwärtigen Interesses, weil selbst dann, wenn die Alltagssprache solche Begriffe enthält, der Begriff einer bloßen Handlungsdankbarkeit in letzter Zeit im Wissenschaftsdiskurs so sehr außer Gebrauch gekommen ist, dass kein Wissenschaftler den Begriff der Dankbarkeit in diesem Sinne mehr zu nutzen scheint. Somit fällt dieser Sachverhalt schwerlich unter das, was wir hier begriffliche Auseinandersetzung nennen.

Die Tugendethik hat nicht nur die Aufmerksamkeit von der Dankbarkeit als einem nicht-emotionalen moralischen Handeln zur Dankbarkeit als einem emotionalen Moralurteil verlagert, sondern auch den Schwerpunkt der auf die Dankbarkeit bezogenen Forschung von der episodischen Dankbarkeit zur Dankbarkeit als Charakterzug. Die Grundbegriffe der Tugendethik sind offenkundig Tugend und Laster. Tugenden und Laster werden als eingelebte (stabile und konsistente) Charakterzustände (Griechisch: hexeis) verstanden, die mit einem Set von Zuwendungen, Emotionen, Wünschen, Verhaltens- und Ausdrucksweisen in bestimmten (charakteristischen und unterscheidbaren) menschlichen Lebensbereichen $\mathrm{zu}$ tun haben, das moralisch lobens- oder tadelnswert ist. Allerdings sind nicht alle diese Komponenten für die Zuerkennung einer Tugend (beziehungsweise eines Lasters) unbedingt erforderlich. Eine Person, die bewegungsunfähig und nicht in der Lage ist, direkt zu handeln oder sich auszudrücken, kann dennoch die Tugend des Mitleids besitzen, wenn sie über die entsprechende Emotion (Kummer über das unverdiente Unglück einer anderen Person) in Zusammenhang mit dem Wunsch zu helfen verfügt (Kristjánsson 2013, Kap. 1.5). Gleiches würde wohl auch, dem Verständnis der Tugendethik nach, für Dankbarkeit als einer potenziellen Tugend gelten. Die Person P könnte für dankbar gehalten werden - ganz und vorbehaltlos -, obwohl P aus irgendeinem Grund nicht fähig ist oder nicht die Möglichkeit hat, dieser Emotion in einer Handlung Ausdruck zu verleihen.

Der in der jüngsten philosophischen Literatur maßgeblich gewordenen Auffassung zufolge ist Dankbarkeit eine emotionale „Neigung“ [,,proneness“]. Gleiches gilt auch für einen beträchtlichen Teil der psychologischen Literatur (Roberts 2004, S. 68). Ein Grund dafür, dass Moralphilosophen und Psychologen stärker an charakterbezogener als an episodenbezogener Dankbarkeit interessiert sind, findet sich in Aristoteles' allgemein bekannter Bemerkung, dass wir Personen nicht für ihre Emotionen als episodischer Ereignisse tadeln oder loben - zum Beispiel dafür, einfach wütend zu sein -, sondern als eingelebter Charakterzustände, die Tugenden oder Laster bilden (Aristoteles, NE 1105b-1106a). Aristoteles' Auffassung zufolge, der ein Großteil der gegenwärtigen tugendbasierten Emotionstheorie beipflichtet, können wir nicht die Erfahrung von Emotionsereignissen steuern, wenn die entsprechenden emotionalen Veranlagungen, sie zu erfahren, erst einmal vorhanden sind. Aber wir sind gemeinsam (auch gemeinsam mit unseren Ethikerziehern) für die Schaffung unserer Dispositionen verantwortlich, indem wir zugelassen haben, dass sie allmählich von uns Besitz ergreifen, ebenso wie ein Alkoholiker dafür verantwortlich gemacht werden kann, einer geworden zu sein, obwohl er sich bei fortgeschrittenem Alkoho- 
lismus nicht mehr dafür verantwortlich machen lässt, wenn er sich betrinkt (Oakley 1992).

Falls nun das, womit wir - ob nun von einem ethischen oder einem psychologischen Gesichtspunkt ausgehend - befasst sind, Dankbarkeit als Charakterzug (und als potenzielle Tugend) ist, scheint es im Interesse begrifflicher Klarheit zu sein, dass wir uns darauf als „Dankbarkeit“ [,gratefulness“] beziehen, und zwar im Gegensatz zu „Dank“ [,gratitude“], verstanden im Sinne einer Episode - so wie es logischer wäre, uns auf die aristotelische Tugend wirklichen Stolzes (,pride“) als „Stolzsein“ [,,pridefulness“"] zu beziehen. Problematisch ist dabei nur, dass (a) diese Unterscheidung von der Alltagssprache ersichtlich nicht gestützt wird und (b) „Dankbarkeit“ [,gratefulness“] von einigen Philosophen als terminus technicus eingeführt wird, um einen subtilen Unterschied zu einem anderen Charakterzug zu bezeichnen, dem der Verbundenheit [,,thankfulness"] . ${ }^{3}$ Es scheint, als ob wir uns hier mit den Unwägbarkeiten der Umgangssprache bescheiden und akzeptieren müssen, dass das Substantiv „Dankbarkeit“ und das Adjektiv „dankbar“ sich in vernünftiger Weise nutzen lassen, um sowohl eine spezifische episodische Emotion als auch einen spezifischen emotionalen Charakterzug oder eine Tugend im Sinne der aristotelischen Tradition zu bezeichnen (Nisters 2012).

Wir sollten uns jedoch lieber darüber klar werden, auf welchen der beiden Begriffe wir uns beziehen, wenn wir die Termini „,Dankbarkeit“ und „,dankbar“ benutzen. Als Verfechter begrifflicher Genauigkeit haben hier Philosophen gegenüber den Psychologen tendenziell die Oberhand (Roberts 2004). Dennoch ist auch ein Teil der neueren psychologischen Literatur dahingehend hinreichend deutlich, dass im Mittelpunkt der Aufmerksamkeit der Begriff der Dankbarkeit als einer emotionalen Disposition steht (McCullough et al. 2001, 2002). Die beiden wesentlichen Instrumente zur Messung von Dankbarkeit sind mit Positionen wie „In meinem Leben gibt es so vieles, für das ich dankbar bin“ oder „Scheinbar habe ich nie das Glück, das andere haben“ auf unser Dispositionsverständnis ausgerichtet. Watkins und Mitarbeiter vermuten, dass Personen mit dem Charakterzug der Dankbarkeit dafür empfänglich sind, episodische Dankbarkeit zu erfahren: je stärker der Charakterzug, desto größer die Wahrscheinlichkeit, dass sich Dankbarkeit ereignet (Watkins et al. 2003). Sie vertreten die Annahme, dass diese Beziehung deshalb besteht, weil bei den Menschen, die von ihrer Anlage her dankbar sind, der Schwellenwert dafür, Dankbarkeit zu erfahren, geringer ist. Darüber hinaus haben einige Psychologen auf die Bedeutung der Unterscheidung zwischen Dankbarkeit als Zustand und Dankbarkeit als Charakterzug sowie auf das Erfordernis verwiesen, beide zu analysieren (Watkins et al. 2003; Tsang et al. 2012).

Die Definition von Dankbarkeit in der Art der positiven Psychologie als Charakterstärke beseitigt offensichtlich jede Zweideutigkeit zwischen Episode und Zustand, weil das Emotionsereignis nicht als Charakterzustand zählt (Peterson, Seligman 2004). Dennoch ist auch die positive Psychologie am Begriff der Dankbarkeit als einer spezifisch flüchtigen und temporären Emotion interessiert. Als zum Beispiel Frederickson und Mitarbeiter den Versuch unternahmen, bei Personen den broaden-

\footnotetext{
3 Anmerkung des Übersetzers: Hier wird „gratitude“ nicht, wie die bisherigen Ausführungen nahelegen könnten, mit „Dank“ übersetzt, sondern durchgängig mit „Dankbarkeit“.
} 
and-build-Effekt durch Erzeugung positiver Gefühle (wie jenem der Dankbarkeit) zu aktivieren (zum Beispiel durch das Vorspielen von Filmausschnitten unbeschwerten Inhalts), anschließend deren Performanz oder Funktion (zum Beispiel ihre Kreativität) zu messen oder sie zu bitten, Fragebögen hinsichtlich ihres Wohlbefindens auszufüllen, war das, was hervorgerufen wurde, vermutlich Dankbarkeit als eine episodische Emotion (Fredrickson 2004 und 2009).

\section{Ein triadischer oder ein dyadischer Begriff?}

Eine bei der philosophischen Analyse von Dankbarkeit geläufige Annahme ist die, dass Dankbarkeit ein triadischer Begriff sei, dessen drei Variablen der Begünstigte, die Gunst und (was für unsere gegenwärtigen Fragen am bedeutsamsten ist) der Begünstigende sind, dem der Begünstigte dankbar ist. Roberts trifft den Hauptgedanken dieser Annahme mit seiner Behauptung, dankbar zu sein bedeute, ,jemandem dankbar zu sein“" (Roberts 2004, S. 63). Er glaubt, dass jene falsch liegen, die behaupten, Dankbarkeit habe eine dyadische Struktur, der entsprechend ein Individuum dankbar für eine Gabe sei, ohne dass es einen Begünstigenden gäbe. Er versichert, ein Begünstigender sei bei der Zuweisung von Dankbarkeit immer, zumindest implizit, mitgemeint. Roberts' Geschichte eines freundlichen Menschen (Carl), der einer Frau einen schweren Sack Kartoffeln vor die Tür stellt, ist ein treffendes Beispiel. Sie dankt ihm für die Gefälligkeit, aber er antwortet: „Danken Sie nicht mir, danken Sie den Kartoffeln!“"Was Carl betrifft, hat Dankbarkeit eine dyadische Struktur. Es gebe einen Empfänger des Wohlwollens, der das Wohlwollen auf das Geschenk selbst bezieht. Ein Erfordernis, einen Begünstigenden ins Spiel zu bringen, bestehe nicht. Roberts hält es für wahrscheinlich, dass Carl einfach zu verstehen geben will, dass er für die Gunst nicht auf Anerkennung aus ist: „Nicht der Rede wert“, „Nichts zu danken“ oder einen anderen Ausdruck von Bescheidenheit hätte Carl äußern können. Allerdings schließt Roberts auch die Möglichkeit nicht aus, dass Menschen, die Dankbarkeit in einem dyadischen Sinne nutzen, ihre eigenen Emotionen missverstehen. Carr stimmt Roberts dahingehend zu, dass die Lockerung der Bedingung des Begünstigenden - der zufolge jemand einfach für etwas dankbar sein kann, ohne jemandem dankbar zu sein - einen schwächeren oder eher abgeleiteten Sinn des Terminus nahelegt, der die entscheidende logische Grammatik von Dankbarkeit vermissen lässt. Es handelte sich dann um einen Sinn, in dem sich das Wort „dankbar“ umstandslos durch „froh“, ,erfreut“ oder „,glücklich“ ersetzen ließe, ohne dass der erforderliche Sinn verlorenginge (Carr 2013).

Triadische Emotions-Charakterzug-Begriffe sind wohlbekannt - zum Beispiel der Begriff der Eifersucht, der sich von dem dyadischen Begriff einfachen Neids unterscheidet (Kristjánsson 2002). Wohlbemerkt müssen für die triadische Struktur eines Begriffs nicht alle beteiligten Seiten eindeutig festgelegt sein. So kann Dankbarkeit auch dann als triadisch gelten, wenn der Begünstigende als eine ungenannte Gruppe von Menschen (,Menschen, die freundlich zu mir waren“) oder selbst als eine unpersönliche Macht, wie etwa das Glück, gilt - solange man annimmt, dass eine begünstigende Instanz am Werke ist. Offenbar ist das Muster von Dankbarkeit als 
einer triadischen Beziehung in der westlichen Kultur die Dankbarkeit in Bezug auf Gott.

Genau genommen muss eine Tätigkeit nicht bei allen beteiligten Seiten angenommen werden. Naito und Mitarbeiter haben die Dankbarkeit von Menschen gegenüber einer nicht-personalen Entität untersucht, die nicht absichtlich eine Gunst erweisen kann - die Natur (Naito et al. 2010). Die Vorstellung der Natur als eines Begünstigenden wirft die Frage auf, ob eine dyadische oder eine triadische Struktur angemessener wäre: Sind wir dankbar für die Wohltaten, die die Natur erweist, und erfassen folglich die Natur als Begünstigenden oder sind wir einfach für die Natur dankbar, und Punkt - erfassen also eine dyadische Struktur von Gunst und Begünstigtem. Logisch gesehen mag an der Dankbarkeit gegenüber einem unbelebten Ding nichts falsch sein - gegenüber einem Holzblock etwa, den ein Schwimmer benutzt, um sich vor dem Ertrinken zu retten. Der Satz ,Ich bin dem Holzblock dankbar, mir das Leben gerettet zu haben“ behält weiter die triadische Struktur von Dankbarkeit. Vermutlich ist dies die Grauzone von Dankbarkeit, den die Entwickler von GQ-6 bewahren wollten, als sie Punkt 6 formulierten: „Viel Zeit kann vergehen, ehe ich Dankbarkeit gegenüber etwas oder jemandem empfinde“ (McCullough et al. 2002). Jedoch muss gesagt werden, dass der Ausdruck ,etwas dankbar sein“ die Grammatik an ihre Grenzen bringt - und uns ist nicht klar, ob er in der Umgangssprache einen Platz hat. (Dies ist ein weiterer Aspekt, den eine sorgsame Untersuchung des Alltagsgebrauchs von Dankbarkeit offenlegen würde.)

Trotz des enormen Wirkungspotentials, den das triadische Verständnis von Dankbarkeit bietet, gibt es Philosophen, insbesondere aber Psychologen, die dieses Verständnis für zu restriktiv halten. Dankbarkeit müsse sich nicht an irgendeinen spezifischen oder unspezifischen, wirklichen oder nur vermuteten Begünstigenden richten, behaupten sie; wir könnten einfach schlechthin dankbar für eine Gunst sein. Der Philosoph McAleer hat die Behauptung, wahre Dankbarkeit könne einen, wie er es nennt, rein ,propositionalen“ Charakter besitzen, beherzt verteidigt. Er verweist hierbei auf Aussagen der Form „A ist dankbar, dass p“, im Unterschied zu gerichteter Dankbarkeit („A ist B dankbar für X“) (McAleer 2012). McAleer macht geltend, dass propositionale und gerichtete Dankbarkeit ,nicht zwei verschiedene Arten von Dankbarkeit sind [...], sondern eine, die in manchen Fällen gerichtet ist, in manchen Fällen nicht.“ Sein Musterbeispiel ist eine Äußerung wie „Washington war dankbar dafür, dass er und viele seiner Leute mit dem Leben davongekommen waren." Dies ist ein etwas unglückliches Beispiel, denn die Äußerung hat - angesichts dessen, was wir über Washington wissen - mit hoher Wahrscheinlichkeit die Form einer Ellipse und lautet im Ganzen: „Washington war Gott dankbar dafür, dass er und viele seiner Leute mit dem Leben davongekommen waren." In der Tat betonen einige Theologen, dass Behauptungen, die eine generalisierte, nicht-gerichtete Dankbarkeit betreffen - zumindest aber Behauptungen der Dankbarkeit für das Leben und dessen Segnungen - die Existenz eines „Gebenden“ beinhalten. „Der Ungläubige, den Dankbarkeit für sein Dasein erfüllt, ist kein Ungläubiger mehr.“ (Tillich 1964, S. 169) Es gibt durchaus gute Gründe für die Vermutung, dass einige der philosophischen Annahmen zugunsten eines triadischen Verständnisses durch ein theologisches Weltbild motiviert sind. Roberts zum Beispiel macht kein Hehl aus seiner Religiosität, was für Steindl-Rast darauf verweist, dass Roberts triadische 
Konzeption „mit der theistischen Theologie“ hinsichtlich eines judeo-christlichen Gottes als letzter Quelle aller Wertigkeit ,eng verbunden“ ist (Steindl-Rast 2004, S. 287).

Während Philosophen das Dyade-Triade-Problem unter Rückgriff auf das ihnen verfügbare Arsenal von Analyseverfahren debattieren, hat es in Kreisen der Psychologie kaum zu einer Theoriediskussionen geführt - mit Ausnahme von McCullough, Kilpatrick, Emmons und Larson, die zu dem Schluss gekommen sind, dass Forscher bisher ,dieses Problem noch nicht in hierreichender Tiefe ausgelotet haben“ (McCullough et al. 2001, S. 256). Dieser Mangel an Tiefenschärfe hat Psychologen jedoch nicht davon abgehalten, in ihrer eigenen empirischen Arbeit ein bestimmtes Begriffsverständnis vorauszusetzen. Algoe, Haidt und Gable folgen dem triadischen Begriffsverständnis, jedoch ohne dies zu begründen (Algoe, Haidt, Gable 2008). Eine Erklärung dafür könnte darin liegen, dass die psychologische Forschung in erheblichem Maße dadurch motiviert ist, interpersonale Beziehungen zu verbessern. Dieses Motiv ist bei Bartlett und DeSteno erkennbar, die darauf verweisen, dass Dankbarkeit dabei hilft, soziale Beziehungen zu schaffen und zu erhalten, indem sie Gegenseitigkeit und Vertrauen zwischen Begünstigendem und Begünstigtem befördert (Bartlett \& DeSteno 2006). (...)

Das überzeugendste Argument gegen ein dyadisches Verständnis von Dankbarkeit - und zugleich ein Argument gegen McAleers These, dass gerichtete und generalisierte (propositionale) Dankbarkeit einfach Vorstellungen ein und desselben Begriffs sind - lautet, dass die Umgangssprache bereits einen unabhängigen Begriff dessen bereithält, was einige Theoretiker als generalisierte Dankbarkeit bezeichnen möchten. Dieser Begriff nennt sich Wertschätzung [appreciation] und ist eindeutig dyadischer Natur. Er bezeichnet die Beziehung zwischen einer Person und einer Sachlage, in der die Person den Wert und die Bedeutung dieser Sachlage anerkennt und an diese emotional gebunden ist. Fagleys empirische Studie (in der sie Adlers und Fagleys Wertschätzungs-Skala nutzt) gibt an, die Unterschiede in der Lebenszufriedenheit nach der Einbeziehung anderer Faktoren, einschließlich der Dankbarkeit, zu erklären (Fagley 2012; Adler/Fagley 2005). Genauer gesagt erklärt in Fagleys Studie Wertschätzung $11 \%$ der Lebenszufriedenheit jenseits von Dankbarkeit, wobei die Messung mittels GQ-6 erfolgte (was noch bemerkenswerter ist, da in GQ-6 bereits drei Punkte Wertschätzung als generalisierte Dankbarkeit beinhalten). Fagley schließt, dass Wertschätzung mehr als bloße Dankbarkeit ist.

Adler und Fagley behaupten, dass Wertschätzung durch ein bedauerliches Ereignis ausgelöst werden kann: Wenn man sieht, wie ein Tornado das Haus eines anderen zerstört, wird man möglicherweise dankbar dafür sein, dass das eigene Haus unversehrt geblieben ist. Hier gibt es offenkundig keinen Begünstigenden, dem man dafür dankbar sein oder den man dafür wertschätzen kann (Adler/Fagley 2005). Interessanterweise schlagen Adler und Fagley vor, Dankbarkeit als ein untergeordnetes Merkmal der Wertschätzung zu betrachten. Darüber hinaus sind sie der Auffassung, dass Dankbarkeit eigens bei Anerkennung einer Gunst hervorgerufen wird und diese Anerkennung mit einem Gefühl der Verbundenheit gegenüber einem Begünstigenden einhergeht, dem sich diese Gabe verdankt. Im Unterschied zur Betonung der begrifflichen Unterscheidung zwischen Wertschätzung und Dankbarkeit zeigt ein Vergleich, den Wood, Maltby, Stewart und Joseph zwischen GRAT, GQ-6 
und der Wertschätzungsskala durchgeführt haben, dass Dankbarkeit und Wertschätzung ein einzelfaktorielles Persönlichkeitsmerkmal bilden (Wood et al. 2008). Das heißt, dass bei diesen Bewertungen von Wertschätzung und Dankbarkeit dieselbe Sache gemessen wird, nicht aber zwei gesonderte Begriffe. Daraus schließen die Autoren, dass all diese Maßstäbe ,denselben inhärenten Begriff messen, nämlich eine durch Dankbarkeit und Wertschätzung geprägte Einstellung zum Leben“ (623). Auch hier geht einiges durcheinander, sowohl empirisch als auch begrifflich.

Es überrascht, dass nicht weitere Sozialwissenschaftler den Weg gegangen sind, den Lambert, Graham und Fincham eingeschlagen haben, nämlich zu versuchen zu ergründen, was gewöhnliche Menschen mit Dankbarkeit meinen. Das scheint ein für Psychologen sachdienliches Unterfangen zu sein, wenn sie vermeiden wollen, Dankbarkeit auf eine Weise zu operationalisieren, die den Probanden faktisch die Bedeutung von Dankbarkeit oktroyiert (Lambert et al. 2009). Die Prototypenanalyse von Lambert und Mitarbeitern, die in einem späteren Abschnitt genauer untersucht wird, verweist darauf, dass der alltagssprachliche Begriff von Dankbarkeit üblicherweise Wertschätzung als Subfaktor einschließt und folglich ein Begriff generalisierter Dankbarkeit ist. Letztere definieren sie als ,die Emotion oder den Zustand, der aus dem Bewusstsein und der Wertschätzung dessen folgt, was einem selbst wertund bedeutungsvoll ist" (Lambert et al. 2009, S. 1194). Prototypenanalysen haben allerdings ihre Grenzen. Sie bieten ihren Probanden keine sorgsam ausgearbeiteten Vignetten für die Untersuchung von Intuitionen über Grenzfälle. Es wäre deshalb voreilig zu schließen, dass ein dyadischer Begriff der Dankbarkeit von der Alltagssprache durchgängig gestützt wird. Was erklärt - oder wegerklärt - werden müsste, ist Roberts' beharrliche Intuition, bei Dankbarkeit handele es sich, im Unterschied zur bloßen Wertschätzung, um eine ,zutiefst soziale Emotion“, zu der über eine Gunst hinaus mindestens zwei Akteure gehören, und dass Menschen dann, wenn sie die Bezeichnungen der beiden Emotionen der Dankbarkeit und der Wertschätzung verwechseln, ihre affektiven Zustände falsch markierten (Roberts 2004, S. 65; McCullough et al. 2001, S. 256).

Um schließlich zu den Philosophen und Theologen zurückzukehren: Walker erklärt, wir nutzten das Wort ,gratefulness“ für den dyadischen Begriff, jedoch das Wort ,gratitude“ für den triadischen Begriff (Walker 1980-81). Steindl-Rast behauptet, der triadische Begriff sollte als ,,thankfulness“ bezeichnet werden, interpersonale Dankbarkeit hingegen als „gratefulness“ (Steindl-Rast 2004, S. 286). Obwohl für diese Unterscheidungen verständliche Begründungen vorgelegt werden, ist dennoch einzugestehen, dass terminologische Unterschiede dieser Art in der Tat den zunehmenden Eindruck der Konfusion in diesem Forschungsbereich weiter verstärken.

Das Ergebnis dieses Abschnitts lautet, dass es zwei Verständnisse von Dankbarkeit gibt, die in der Literatur um Anerkennung ringen. Ferner sind diese Verständnisse so verschieden voneinander, dass sie - mehr als nur verschiedene Verständnisse zu sein - als verschiedene Begriffe von Dankbarkeit zu begreifen sind. Dem einen zufolge ist Dankbarkeit die wesentlich soziale Emotion einer einstellungsbezogenen Beziehung eines Begünstigten zur Gunst eines Begünstigenden. Diese ist als ,triadische Dankbarkeit" bezeichnet worden. Dem zweiten Begriff zufolge ist Dankbarkeit die gewohnheitsmäßige Aufmerksamkeit auf die Vorteile, die das Leben unter Ab- 
sehung irgendeines bestimmten Begünstigenden bereithält, sowie die Wertschätzung dieser Vorteile. Er wird hier „dyadische Dankbarkeit“ genannt.

\section{Die Bedingung der Supererogation, die Bedingung der Intentionalität oder keines von beiden?}

Einige Theoretiker behaupten, dass Dankbarkeit, um angemessen zu sein, nicht nur eine triadische Struktur benötigt, die auf einen bestimmten Begünstigenden gerichtet ist, sondern auch verlangt, dass der Begünstigende supererogatorisch gehandelt hat. Er ist also damit, dass er dem Empfänger eine Gunst erwiesen hat, über das hinausgegangen, was die Pflicht gebietet. So macht etwa Simmons geltend, dass Dankbarkeit immer einen erheblichen Verzicht beinhaltet und nicht angemessen ist, wenn mit der Gunst allein Handlungen verbunden sind, die der Pflicht genügen oder eine Schuldigkeit sind (Simmons 1979). Man betrachte auch Roberts' Begriffsbedingung Nr. 3: „Mit der Zuerkennung von $\mathrm{X}$ ist $\mathrm{S}$ über das hinausgegangen, was er mir schuldet“" (Roberts 2004, S. 64). Er schildert das Beispiel einer Person, die (in unangemessener Weise) Dankbarkeit gegenüber ihrem Arzt dafür empfindet, eine zutreffende Diagnose erhalten zu haben - nach einer langwierigen Zeit mehrerer Fehldiagnosen. Roberts zufolge, ,verhielt sich der Arzt nicht großzügig, sondern überlegte nur für kurze Zeit, was mir fehlen könnte. Er führte eine routinemäßige Biopsie durch, setzte die Antibiotika ab und schrieb der Krankenkasse eine Rechnung, die es in sich hatte“ (Roberts 2007, S. 24). Dankbarkeit (und ihre Entsprechung im Falle von Großzügigkeit) muss für Roberts über das hinausgehen, was gerecht oder erwartbar ist und hat vollends wohlwollend und unverdient zu sein.

Roberts kann mittels seiner bekannten allgemeinen Emotionstheorie, der zufolge Emotionen ,anliegenbasierte Interpretationen“ [concern-based construals] sind, erklären, was falsch läuft, wenn dieser Begriffsbedingung (und den anderen von ihm aufgestellten) nicht entsprochen wird: Der Begünstigte verkennt dann die Situation als eine der Dankbarkeit oder macht eine entsprechende Fehlzuschreibung. In solchen Fällen wird Dankbarkeit tatsächlich empfunden, ohne dass sie angemessen wäre (Roberts 1988; Roberts 2004, S. 62). Steindl-Rast hat das Supererogationsmerkmal von Dankbarkeit etymologisch erklärt: Der Wortstamm von ,gratitude“ ist „grati“ und verweist darauf, dass etwas ,gratis“ gegeben wird, unverdient (SteindlRast 2004, S. 284). „Wir sind daher dankbar“, so bemerkt gleichermaßen Walker, „für das, was wir als etwas betrachten, das über unsere Rechte oder Anrechte hinausgeht“ (Walker 1980-81, S. 48). Abgesehen von diesen etymologischen Betrachtungen behauptet McConnell, dass Menschen denen, die sie begünstigen, selbst dann dankbar sein können, wenn diese nur den mit ihrer Tätigkeit verbundenen Pflichten nachkommen (McConnell 1993). Darüber hinaus sei kein spezieller Verzicht oder Aufwand als unerlässliche Bedingung für Dankbarkeit anzunehmen. Jemand könne auch mit dem Geringen, das er aufwendet, hilfreich sein.

Psychologen führen die kompromisslose Supererogationsbedingung selten ins Feld - mit der Ausnahme von Emmons, der ihr prinzipiell zuzustimmen scheint: „Unverdienter Vorzug ist der Eckpfeiler von Dankbarkeit. Die dankbare Person anerkennt, dass sie nichts dafür getan hat, die Gabe oder Gunst zu erwerben“ (Emmons 
2004a, S. 5). Man sollte jedoch in Betracht ziehen, dass diese Bedingung in Emmons' empirischer Arbeit ersichtlich keine besondere operative Funktion besitzt. Nicht einmal die etablierte psychologische Literatur betrachtet sie als potenzielle Intensitätsvariable (der zufolge Menschen zwar Dankbarkeit auch ohne Supererogation empfinden können, sie Dankbarkeit aber tiefer empfinden, wenn der Begünstigende als jemand erscheint, der etwas getan hat, das über die bloße Pflichterfüllung hinausgeht (McConnell 1993, S. 16)). Dass Psychologen diese Bedingung selten in Betracht ziehen, ist vielleicht in ihrer Präferenz des dyadischen Verständnisses von Dankbarkeit begründet (siehe den vorherigen Abschnitt), während die Bedingung der Supererogation üblicherweise im Zusammenhang eines triadischen Verständnisses aufgestellt wird.

Obwohl wir dies in einer empirischen Untersuchung der Begriffsverständnisse von Akteuren im Kontext alltagssprachlicher Zusammenhänge noch aufzeigen müssen, ist es doch wahrscheinlich, dass die Bedingung der Supererogation mit den Intuitionen der meisten kompetenten Sprecher im Widerspruch steht. Im Grunde genommen verletzten jene Fälle, die für eine angemessene Dankbarkeit paradigmatisch zu sein scheinen - die Dankbarkeit, die zum Respekt gegenüber den Eltern gehört, die Dankbarkeit gegenüber einem Rettungsschwimmer, der einen vor dem Ertrinken gerettet hat -, diese Bedingung auf eklatante Weise (McConnell 1993, S. 16).

Während Roberts' Theorie ,anliegenbasierter Interpretationen“ Fälle kognitiver Dissonanz gut erklären kann, ließe sich einwenden, dass er sie falsch anwendet, wenn er Individuen ein falsches Bewusstsein zuspricht, die seiner Untersuchung entsprechend fälschlicherweise dort Dankbarkeit erfahren, wo sie „unbegründet“" sei. Womöglich spiegeln sich in Roberts' Verbindlichkeit gegenüber der Auffassung, der zufolge zur Dankbarkeit ein Begünstigender gehört, dessen Tun über das von der Gerechtigkeit im eigentlichen Sinne Geforderte hinausgeht - dessen Tun eine Gabe grundlosen und ganz unverdienten Charakters übermittelt -, eigene christliche Hintergrundüberzeugungen. Roberts' Kernerfahrung und Modell von Dankbarkeit sind die einer wunderbaren Gnade. Diese wird ihm zum Muster einer Dankbarkeit, die sich dadurch auszeichnet, dass sie vom Prinzip her barmherzig über das hinausgeht, was die Gerechtigkeit verlangt. Ungeachtet dieses Vorschlags präsentiert die Bedingung der Supererogation in der Tat eine solch einzigartige Vorstellung von Dankbarkeit, dass wir zu sagen versucht sind, ein Verständnis von Dankbarkeit, das sie als eine begrifflich notwendige Bedingung beinhaltet, bezeichne einen Sonderbegriff von Dankbarkeit.

Eine weitere mögliche Bedingung, die viele Philosophen der Dankbarkeit auferlegen, besteht darin, dass der Begünstigende die entsprechende Gunst dem fraglichen Begünstigten (starke Intentionalitätsbedingung) oder zumindest einem möglichen Begünstigten (schwache Intentionalitätsbedingung) absichtlich erwiesen haben muss (McConnell 1993, Kap. 1). Etwas überraschend scheinen viele Psychologen der Annahme dieser Bedingung beizupflichten - setzt die Intentionalitätsbedingung doch einen triadischen Begriff von Dankbarkeit voraus, während Psychologen oft einen dyadischen Begriff präferieren. Wie wir allerdings sehen werden, gibt es in der psychologischen Literatur einige Unklarheiten über die Natur dieser Bedingung. 
Die Vorstellung, dass man bei guten Folgen ohne gute Absichten keine Dankbarkeit schuldet, geht auf den stoischen Philosophen Seneca zurück (Harpham 2004). In der psychologischen Literatur wird diese Ansicht sehr häufig Heider zugeschrieben (Heider 1958). Obwohl die psychologische Behauptung oftmals als eine offenkundige begriffliche Bedingung formuliert wird (so zum Beispiel: „Dankbarkeit entsteht, wenn ein Individuum [Begünstigter] bemerkt, dass eine andere Person [Begünstigender] oder eine Quelle [zum Beispiel Gott, Glück, Schicksal] in der Absicht gehandelt hat, das Wohl des Begünstigten zu mehren“), kann man, wenn man die Bedingung analysiert, feststellen, dass sie nichts anderes als eine Intensitätsvariable ist (Fredrickson 2004, S. 150). So nehmen McCullough und Tsang an, dass „Menschen am wahrscheinlichsten dann Dankbarkeit empfinden“, wenn der „Aufwand an Mühe zu ihren Gunsten eher absichtsvoll als zufällig erbracht worden zu sein scheint" (McCullough/Tsang 2004, S. 125-126). Gleichermaßen zeigt Tsangs empirische Untersuchung, dass Menschen erheblich mehr Dankbarkeit empfinden, wenn der Begünstigende wohlwollende Absichten hegt (Tsang 2006). Dieser Befund erinnert an die früheren Ergebnisse von Tesser, Gatewood und Driver, die nachgewiesen hatten, dass Gefühle der Dankbarkeit eine Funktion der Wahrnehmung der Absichten des Begünstigenden seitens des Begünstigten, des Aufwands des Begünstigenden für die Gunst und des Wertes der Gunst für den Empfänger ist (Tesser, Gatewood, Driver 1968).

Es ist eine Sache zu sagen, dass Absicht eine notwenige Bedingung angemessener Dankbarkeit ist, eine ganz andere ist es hingegen, dass es die Dankbarkeit mehrt, wenn sie von einer Absicht begleitet wird (womit offenbleibt, dass Dankbarkeit auch in Ermangelung der entsprechenden Absichten des Begünstigenden noch angemessen empfunden werden kann). Die psychologische Literatur scheint einer Entscheidung zwischen diesen beiden Auffassungen auszuweichen. Ist zum Beispiel von Wood, Joseph und Maltby eine begriffliche oder eine empirische Unterscheidung getroffen worden, als sie behaupteten, Dankbarkeit ,folgt auf eine Hilfe, die aufwendig, wertvoll und altruistisch gemeint ist“" (Wood, Joseph, Maltby 2008b)? Der Grund für diese Unklarheit lässt sich auf Heiders Werk zurückverfolgen, in dem er behauptete, Intentionalität sei der bedeutsamste Faktor, um festzustellen, ob man Dankbarkeit empfindet oder nicht (Heider 1958). Heider behauptete, ein Begünstigter empfinde nur dann wirkliche Dankbarkeit, wenn er die Gunst als etwas erkennt, das durch den Begünstigenden absichtsvoll erwiesen wurde. Die Unklarheit besteht jedoch in der Formulierung ,wirkliche Dankbarkeit“. Ist diese begrifflich gemeint (sodass Dankbarkeit in Ermangelung empfundener Absicht vernünftigerweise nicht empfunden werden kann) oder empirisch (sodass Dankbarkeit auch in diesem Falle empfunden werden kann, allerdings in solch schwachem Maße, dass sie nicht als bedeutsam zählt)?

Jene Theoretiker (ob nun Psychologen oder Philosophen), die entweder davon ausgehen, dass Dankbarkeit ohne Anerkennung von Intentionalität vernünftigerweise nicht empfunden, oder doch nur in unbedeutend schwachem Maße empfunden werden kann, bringen normalerweise eine andere Emotion ins Spiel, um solche Fälle zu erklären, in denen das Gefühl besteht, eine Entschädigung leisten oder eine Gabe erwidern zu müssen, ohne dass Dankbarkeit vorkommt - nämlich Verbindlichkeit [indebtedness] (Berger 1975, S. 299). So argumentiert Tsang auf Grundlage ihrer 
empirischen Studie, dass es, obgleich Dankbarkeit und Verbindlichkeit von einigen Forschern miteinander vermengt werden, zwischen beiden eine Reihe auffallender theoretischer und empirischer Unterschiede gibt. Ein Unterschied bestehe darin, dass Dankbarkeit eine „Emotion mit positiver Wertigkeit“ ist, während Verbindlichkeit „von negativen Gefühlen wie Unbehagen und Unwohlsein begleitet wird“ (Tsang 2006, S. 200). Tsang hat die Wirkungen eines eigennützig gegebenen Gefallens untersucht und festgestellt, dass die Empfänger dabei kaum Dankbarkeit empfunden hatten. Andererseits bleiben Gefühle der Verbindlichkeit konstant und sind von Hilfsintentionen unabhängig. Ihre Behauptung einer wesentlichen Unterscheidung zwischen Dankbarkeit und Verbindlichkeit wäre offensichtlich überzeugender gewesen, wenn sie den Befund erbracht hätte, dass Empfänger gegenüber dem Geber des eigennützig gegebenen Gefallens keinerlei Dank empfunden hätten. Dass dies nicht der Fall war, scheint zumindest auf eine Überschneidung zwischen beiden Begriffen hinzudeuten. (...)

Statt allein danach zu fragen, ob die Absicht des Gebers wahrnehmbar vorliegt oder nicht, lädt Tsangs Untersuchung auch dazu ein, sich mit dem Inhalt zu beschäftigen. Was wäre, wenn die Absicht nicht nur eigennützig und nicht wohlwollend, sondern unverhohlen niederträchtig ist? Gesetzt den Fall, ein entfernter Verwandter habe Sie testamentarisch zu einem der Hauptbegünstigten seines Erbes gemacht, wobei Ihnen bewusst ist, dass der Begünstigende damit die Absicht verband, der Beziehung zwischen Ihnen und den übrigen Verwandten zu schaden. Wäre es dennoch begrifflich möglich, Dankbarkeit zu empfinden - oder Verbindlichkeit? Fitzgerald kappt die Verbindung zwischen wohlwollender Absicht und Dankbarkeit völlig, indem er behauptet, Menschen könnten Dankbarkeit in Reaktion selbst auf jene empfinden, deren Absicht es möglicherweise gewesen ist, Unheil zu stiften (Fitzgerald 1998). Man betrachte hier als einen Grauzonenfall Roberts' Beispiel des Dalai Lama, der den Chinesen, obwohl deren Absichten böswillig sind, seinen Dank dafür zum Ausdruck bringt, ihm und anderen die Gelegenheit zu bieten, Geduld zu praktizieren (Roberts 2007). An dieser Stelle sollte beachtet werden, dass die buddhistische Auffassung Teil eines umfassenderen ethischen und metaphysischen Systems ist, demzufolge Individuen jenen zu danken haben, die sie in Versuchung führen. Verschafft ihnen dies doch die Möglichkeit der Selbstprüfung und der Betrachtung darüber, was einen Einzelnen weiterhin an die vergängliche und kurzlebige Welt bindet. Nur durch Auflösung dieser Bindungen kann eine Person Erleuchtung erlangen. Die Absicht des anderen ist ohne Bedeutung. Entscheidend ist (und dafür kann man folglich dankbar sein), dass der andere den Weg zur Erleuchtung aufzeigt. Im Gegenzug sollte dann, wenn eine Person etwas gibt, zu dem ein Anderer eine Bindung entwickeln kann, der Empfänger für die Gabe nicht dankbar sein. Dem Empfänger wird damit nämlich eine Falle gestellt. Während Roberts dieses Beispiel als Sonderfall von Dankbarkeit gilt, steht dieser doch in völligem Einklang mit einer buddhistischen Weltauffassung, der zufolge Menschen denen dankbar sein sollen, die ihnen den „Weg“ aufzeigen (was die einzig wahre Gunst wäre), ganz gleich, wie sie das tun.

So weit wie Fitzgerald, der die Verbindung zur Dankbarkeit kappt, geht McConnell nicht. Er richtet die Aufmerksamkeit hingegen auf die komplexen Zusammenhänge, die eine Rolle spielen, wenn man den Handlungen eines Begünstigen- 
den Intentionalität zuschreibt (McConnell 1993). Obwohl sich theoretisch zwischen Dankbarkeit und Manipulationsversuchen, einen Begünstigten in die Schuld eines Spenders zu bringen, unterscheiden lässt, kann man doch Dank empfinden, ohne zu wissen, ob eine Gabe wirklich grundlos angeboten wurde. Vielleicht ist ein allgemeiner Bestandteil von Dankbarkeit das Vertrauen in die guten Absichten dessen, der gibt, eine Leistung, die eine bestimmte Art Mut oder Vertrauen abverlangt, die dem Mut des Begünstigenden korrespondiert, eine Gabe anzubieten, die letztlich abgelehnt werden kann. Roberts bezieht sich auf diese Allianz zwischen demjenigen, der die Gabe stiftet, und deren Empfänger als ,eine Art tugendbasiertes gegenseitiges Übereinkommen“ [,,a kind of meeting of the minds in virtue"] (Roberts 2007, S. 15).

McConnell bezweifelt auch, ob Eigeninteresse in jedem Fall ein Ausschlusskriterium ist, und verweist darauf, dass Stolz oder Heldentum mit Zwecken einhergehen kann, die anderen Menschen dienen (McConnell 1993, S. 24). Bedeutsam für die Frage nach der Absicht, anderen zu helfen, ist auch die gemeinhin angeführte Feststellung, dass Menschen jenen dankbar sein können, denen sie selbst nützen. Lehrer, Krankenschwestern und Ärzte können Schülern und Patienten dafür dankbar sein, ihnen die Möglichkeit zu geben, ihr Können zu praktizieren. Angesichts dessen, dass Schüler und Patienten normalerweise nicht beabsichtigen, ihren Erziehern, Ärzten oder ihrem Pflegepersonal etwas Gutes zu tun, ist Dankbarkeit vielleicht nicht so eng an die beabsichtigte Großzügigkeit eines Begünstigenden gebunden, wie einige Untersuchungen nahelegen. Erneut ist zu betonen, dass weitere Untersuchungen zum alltagssprachlichen Verständnis nicht nur nützlich, sondern unerlässlich sind, wenn man diesen Fragen mittels empirischer Forschung nachgeht.

\section{Wirkliche Gunst - oder bloß beabsichtigte?}

Eine Kontroverse scheint sich abzuzeichnen, die die Natur der empfundenen Gunst betrifft, die vorhanden sein muss, ehe Dankbarkeit entstehen kann. In den meisten Darstellungen wird problemlos davon gesprochen, dass der Begünstigte für die erwiesene Gunst oder Hilfeleistung dankbar ist, als ob die Gunst immer schon vorhanden sein muss. Es ist aber vielleicht hinreichend, dass die Gunst seitens des Begünstigenden nur beabsichtigt war, obwohl sie letztlich nicht erwiesen werden konnte - man beachte das Sprichwort: „Allein der gute Wille zählt!“ McConnell hat darauf verwiesen, dass dann, wenn jemand versucht, einem anderen einen erheblichen Gefallen zu tun, damit aber scheitert, dennoch Dankbarkeit geschuldet sein kann (McConnell 1993, S. 44). Als Beispiel dafür, dass die Gunst nicht erwiesen werden konnte, schildert er den Fall eines Arbeitskollegen, der einen anderen für eine Auszeichnung vorschlägt, der andere aber die Auszeichnung nicht erhält und auch keinen merklichen Nutzen aus dem Vorschlag ziehen kann. McConnells Darstellung zufolge ist Dankbarkeit in diesem Fall dennoch eine angemessene Reaktion. Von daher beinhaltet sein Verständnis von Dankbarkeit die Bedingung, dass „die Person, der Dankbarkeit geschuldet ist, einen Nutzen gestiftet oder mit großen Mühen oder Opfern versucht hat, einen beträchtlichen Nutzen zu erbringen“ (McConnell 1993, S. 44). Die Vorstellung, dass man für eine Gunst Dank empfinden 
kann, die letztlich nicht erwiesen werden konnte, ist ein Gedanke, der langsam auch in die Psychologie Eingang gefunden hat, wobei einige Autoren eine nicht erwiesene Gunst in ihre Arbeitsdefinitionen eingefügt haben. So wurde Dankbarkeit als eine positive Emotion verstanden, die eine Person empfindet, wenn ihr eine andere Person absichtlich etwas Wertvolles gegeben oder zu geben versucht hat (Bartlett/ DeSteno 2006; McCullough et al. 2001; McCullough/Tsang 2004). Diese Art der Definition bestätigt, dass es zuweilen wirklich der gute Wille ist, der zählt.

In gleicher Weise ließe sich auch vermuten, dass man dankbar für eine beabsichtigte Gunst ist, die man letztlich selber gar nicht für wertvoll hält. Man stelle sich zum Beispiel vor, ein Begünstigender unternehme erhebliche Anstrengungen, einem Begünstigten etwas zu schenken. Der Begünstigende beabsichtigt mit dieser Gabe, die für ihn eine erhebliche Ausgabe bedeutet, dem Begünstigten etwas Gutes zu tun. Ihm ist allerdings unbekannt, dass der gemeinte Begünstigte die Gabe nicht als wertvoll oder nutzbringend betrachtet. Damit ist eine Situation entstanden, in der es (in der Wahrnehmung des Empfängers) keinen wirklichen Nutzen gibt; wohl aber bleibt es bei einem beabsichtigten Nutzen. Bedeutet dies, dass keine Dankbarkeit geschuldet ist, oder sollte der [[scheinbar]] Begünstigte dennoch Dank für die Bemühungen des [[scheinbar]] Begünstigenden empfinden? Wenn es wirklich der Wille ist, der zählt, dann ist in diesem Szenario sicherlich ein gewisses Maß an Dank geschuldet. Allerdings werden Situationen wie diese in psychologischen Betrachtungen zur Dankbarkeit normalerweise übergangen.

Ebenfalls noch nicht diskutiert zu sein scheint die Frage, ob man (vernünftigerweise) stellvertretend Dankbarkeit empfinden kann, wenn die Gunst nicht einem selbst gilt. Man betrachte zum Beispiel die Situation, in der ein Begünstigender einem jungen Mann Geld schenkt. Dieses Geld wird als eine wertvolle Unterstützung für den jungen Mann betrachtet, und dieser ist außerordentlich erfreut über sein neues Vermögen. Die Mutter des jungen Mannes bemerkt, wie glücklich und dankbar ihr Sohn ist und beginnt, dem Begünstigenden gegenüber ebenfalls Dankbarkeit zu empfinden. In diesem Szenario ist eine Gunst erwiesen worden, was direkt zur Dankbarkeit seitens des Begünstigten und indirekt zur Dankbarkeit seitens einer dritten Partei geführt hat. Empfindet die Mutter tatsächlich Dankbarkeit gegenüber dem Begünstigenden? Die meisten Menschen wären wahrscheinlich der festen Überzeugung, dass es sich hier um wirkliche Dankbarkeit handelt und diese Art Situation in keiner Weise ungewöhnlich ist. Aber das Szenario passt nicht zur früher erwähnten triadischen Struktur von Dankbarkeit: Wir haben hier einen Begünstigenden, eine Gunst, einen Begünstigten und ein begleitendes drittes Mitglied oder eine Quadratstruktur. Einige Theoretiker könnten daraufhin fragen, warum das dritte Mitglied Dankbarkeit empfinden sollte und warum dies wirkliche Dankbarkeit wäre. Hier ist vorgeschlagen worden, dass Vorteile als kommunale Gewinne betrachtet werden können. So behauptet Roberts, dass der Begünstigende und der Begünstigte in einer Art moralischer Gemeinschaft miteinander verbunden sind und auch der Begünstigende aus dem, was der Begünstigte erhält, einen Nutzen zieht. Es handelt sich um einen gegenseitigen Vorteil (Roberts 2007, S. 11-12). Diese Art von Überlegung könnte auch auf das obige Szenario Anwendung finden: Das dritte Mitglied und der Begünstigte verbindet ein und dieselbe Gemeinschaft, und deshalb erlangt das dritte Mitglied auch das, was der Begünstigte erlangt. Szenarien 
dieser Art sind gewiss nicht ungewöhnlich. In der Literatur haben sie allerdings nur geringes Interesse gefunden.

\section{Positive oder gemischte Emotionen?}

Wie wir in Kürze sehen werden, ist man in Psychologie und Philosophie üblicherweise der Meinung, Dankbarkeit sei eine in einem moralischen Sinne positive Emotion (obwohl weiterhin Meinungsverschiedenheiten über die genaue Natur ihres moralischen Wertes bestehen). Gleiches gilt aber auch für Mitleid, eine wesentlich schmerzliche Emotion. Wenn Psychologen jedoch von positiver Emotion sprechen, dann neigen sie dazu, eine phänomenologisch positive oder positiv gewertete, das heißt angenehme, Emotion zu meinen (Tsang 2006, S. 200). So gesehen ist Mitleid eine negative Emotion, Dankbarkeit aber wird charakteristischerweise für positiv gehalten. In der Tat ist in der jüngsten Psychologie Dankbarkeit von Wood und Mitarbeitern als das „wesentliche positive psychologische Merkmal“ beschrieben worden, von Tsang und Mitarbeitern als eine ,positive emotionale Erwiderung“ und von Emmons und Shelton (die auch Chestertons Bemerkung zitieren, Dankbarkeit erzeuge ,die reinsten, dem Menschen bekannten Freudenmomente“) als ein ,im eigentlichen Sinne bereichernder Zustand“, der grundsätzlich mit Freude und positiver Stimmung verbunden sei (Wood, Joseph, Lloyd, Atkins 2009, S. 43; Tsang et al. 2012, S. 41; Emmons, Shelton 2002, S. 465 und 460). Fredrickson verortet Dankbarkeit eindeutig in den Bereich positiver Emotionen und feiert sie als eine der Emotionen, die erweiternd und aufbauend seien (Fredrickson 2004). Nicht weniger unbeirrbar ist Emerson: „Es ist wichtig, dass Dankbarkeit einen positiven Wert besitzt. Sie bringt ein gutes Gefühl mit sich“ (Emmons 2004a, S. 5). Nisters hat die mit Dankbarkeit verbundene Freude als dreifach beschrieben: als (1) die Freude, eine Gunst zu erwerben, (2) die Freude, die man empfindet, wenn man einem Begünstigenden dankt, und (3) die Freude, eine Gunst zu erwidern (Nisters 2012, S. 73). Auch Roberts vertritt die Auffassung, Dankbarkeit sei überwiegend positiv und betont die Rolle, die sie dabei spielt, Neid (ein Laster), Unmut und Kummer zu dämpfen (Roberts 2004, S. 66-77).

Es ist aber auch behauptet worden, Dankbarkeit stehe mit der negativen Emotion der Verbindlichkeit in Zusammenhang. Greenberg und Westcott haben hervorgehoben, dass Selbsteinschätzungen der Rektionen auf die Hilfsleistungen seitens anderer die Stärke von Gefühlen des Unbehagens möglicherweise unterbewerten (Greenberg, Westcott 1983). Reaktionen auf Hilfen gründen auf Zuschreibungsmustern, die nicht nur segensreich sind: neben denen der Anerkennung der Begünstigungen gibt es auch Gefühle der Frustration, verpflichtet zu sein und als bemitleidet zu gelten. Emmons und Crumpler haben angeregt, dass man, um wirklich dankbar zu sein, für die Gunst eine Dankesschuld empfinden und zugleich wissen müsse, sie niemals (restlos) begleichen zu können (Emmons, Crumpler 2000). Von anderen Autoren ist der Versuch unternommen worden, die positive Emotion der Dankbarkeit und die negative Emotion der Verbindlichkeit voneinander zu trennen (siehe die obige Diskussion zu diesem Thema). Von McCullough und Mitarbeitern stammt die Behauptung, Verbindlichkeit beinhalte Gefühle der Schuldigkeit und eines quä- 
lenden Drangs, die einem erwiesene Gunst zu begleichen, wohingegen Dankbarkeit grundsätzlich positiv und mit anderen positiven psychologischen Merkmalen wie Zufriedenheit und Zuversicht verbunden sei (McCullough et al. 2001).

Watkins, Scheer, Ovnicek und Kolts (2006) knüpfen an Fredricksons Unterscheidung zwischen positiven Emotionen, die wie Dankbarkeit vermeintlich erweitern und aufbauen, und negativen Emotionen wie Verbindlichkeit an, von denen es heißt, sie führen zu beschränkteren Auffassungen, wie etwa der Vorstellung, eine Gunst einer wie-du-mir-so-ich-dir-Mentalität folgend zurückzuerstatten. Sie analysierten diese Unterscheidung, indem sie untersuchten, ob die beiden Begriffe mit unterschiedlichen Handlungstendenzen in Verbindung stehen. Ihre Ergebnisse zeigten, dass dann, wenn Begünstigende für die erwiesene Gunst einen Gefallen erwarten, die Verbindlichkeit zunimmt, während die Dankbarkeit abnimmt. Angesichts dessen haben Watkins und Mitarbeiter behauptet, dass Dankbarkeit und Verbindlichkeit voneinander getrennt werden können. Interessanterweise fanden sie aber auch Belege für eine signifikante Wechselbeziehung zwischen beiden, ein Befund, der in der früheren Arbeit von Tesser, Gatewood und Driver dokumentiert ist (Tesser et al. 1968).

Ob nun Verbindlichkeit als eine Facette von Dankbarkeit oder als eine eigenständige Entität betrachtet wird, so ist doch klar, dass beide untrennbar miteinander verbunden sind. In einer innovativen Studie von Lambert, Graham und Fincham erschien Verbindlichkeit in der Liste mit 52 Schlüsselmerkmalen von Dankbarkeit und wurde durch die alltagssprachlichen Akteure als ein negatives Attribut betrachtet (Lambert et al. 2009). Zu den anderen Merkmalen, die als negativ bewertet wurden, gehören Verpflichtung und Eifersucht oder Neid. Dieser Befund fällt nicht so hart aus wie die Bewertung von Aristoteles, der Dankbarkeit für seine „hochsinnigen“ Vorbilder moralischer Tugend als grundsätzlich belastend und von den eigentlichen Dingen ablenkend einstuft. Dennoch legt der Befund nahe, dass Dankbarkeit für die Öffentlichkeit sowohl positive als auch negative Aspekte besitzt (Aristoteles, NE 1123a-1125a). Dankbarkeit ist vielleicht nicht so durchgängig positiv, wie viele gegenwärtige Theoretiker vermuten. Möglicherweise gründet diese Kontroverse in dem von der Psychologie übervereinfachten Gegensatz von positiven und negativen Emotionen, einem Gegensatz, mit dem Gefühlskomponenten nicht in Einklang stehen (Fredrickson 2004, S. 153). Aristoteles hatte eine ausgewogenere Haltung zur emotionalen Valenz mit seiner Erklärung, dass viele alltägliche Emotionen wie etwa Zorn weder gänzlich angenehm noch schmerzlich, sondern eher gemischt seien (Kristjánsson 2007). So ist Zorn (der im Wesentlichen schmerzlich ist) auch in dem Maße angenehm, als er auf die Möglichkeit zukünftiger Vergeltung verweist (Aristoteles, Rhet. 1378b). In jedem Fall hat der Befund von Lambert und Mitarbeitern erneut hervorgehoben, wie bedeutsam es ist, die Intuitionen alltagssprachlicher Akteure zum Vorschein zu bringen, statt dass man voreilige Schlüsse über die wahre Natur von Dankbarkeit zieht (zum Beispiel ausschließlich angenehm zu sein) (Lambert et al. 2009). 


\section{Moralische Pflicht oder moralische Tugend?}

Es ist nunmehr Zeit, unsere Aufmerksamkeit den Auseinandersetzungen um die moralische Natur von Dankbarkeit zuzuwenden. Im vorliegenden Abschnitt untersuchen wir verschiedene Auffassungen zu der Frage, welche Art moralischen Wert sie darstellt; im Folgeabschnitt beschreiben wir dann verschiedene Ansichten zu der Frage, wie wertvoll sie ist und warum.

Bis zur zweiten Hälfte des zwanzigsten Jahrhunderts wurde Dankbarkeit, angeregt durch Vorstellungen vom göttlichen Gebot und durch deontologische Moraltheorien, vorwiegend als eine moralische Pflicht oder Schuldigkeit betrachtet. Oft wird auf Thomas von Aquin als den geschichtlichen Vorläufer dieser traditionellen Auffassung verwiesen (Emmons 2004a, S. 7; Harpham 2004, S. 25-26). Für Thomas war Dankbarkeit nichts anderes als eine Erweiterung der Pflicht der Gerechtigkeit der Begleichung einer moralischen Schuld, die dadurch erfolgt, dass anderen (einschließlich und vor allem Gott) das gegeben wird, was ihnen zusteht (Hibbs 2009). In den philosophischen Untersuchungen zur Dankbarkeit hat diese Denkrichtung in Bergers früher Abhandlung einen angemessenen Vertreter: Dankbarkeit gilt als etwas, das wir verpflichtet sind zu ,zeigen oder auszudrücken“, womit wir folglich unsere auf Gerechtigkeit gründenden Anliegen ausweisen (Berger 1975, S. 305). Doch Berger anerkannte auch, allerdings eher beiläufig, dass einige der tradierten Weisen, über Dankbarkeit zu sprechen - nämlich ausschließlich im Sinne von Rechten, Unrechten und Pflichten - zu einer ,,unangemessenen Darstellung dessen“ geführt haben, „was es heißt, moralisch zu sein“ (Berger 1975, S. 306).

Später, d. h. nach Berger, sind Philosophen zunehmend von einer auf Pflichten und Rechten gründenden Auffassung von Dankbarkeit zu einer tugendbasierten Auffassung übergegangen: ein Schritt, der mehr oder weniger mit dem Übergang von der Auffassung von Dankbarkeit als Handlung und Handlungsdisposition zu einer solchen von Dankbarkeit als einer Emotion oder einer affektiven Disposition zusammenfiel, der wir in Abschnitt 2 nachgegangen sind. Dass diese beiden Entwicklungen parallel erfolgten, ist nicht verwunderlich. Letztlich sind alle zeitgenössischen Darstellungen von Emotionen als moralisch bedeutsamen Entitäten in der Regel durch das jüngst wiedererwachte Interesse am moralphilosophischen Begriff der Arete oder der Tugendethik angeregt worden (Oakley 1992, S. 39). Vertreter der Tugendethik werden von ihrer Behauptung nicht abzubringen sein, dass frühere Ansätze, Dankbarkeit ethisch zu interpretieren, im Verdacht stehen, Dankbarkeit mit Verbindlichkeit und Wechselseitigkeit zu vermengen. Statt zu fragen, wem Dankbarkeit zu schulden ist - und welche Handlungen als Korrektiv verlangt sind -, sollten wir überlegen, wann wir gute Gründe haben, als tugendhafte Akteure dankbar zu sein (Fitzgerald 1998). Während wir unsere Verpflichtungen auch dann erfüllen können, wenn wir nicht mit dem Herzen dabei sind, untergraben solche Beispiele das, worum es bei der Dankbarkeit wirklich geht. In der Tat klingt eine „Pflicht, dankbar zu sein, [...] wie ein Witz“ (Card 1988).

Seinen entschiedensten Ausdruck findet dieses neue Verständnis des ethischen Werts von Dankbarkeit in Wellmans Abhandlung, in der er ausdrücklich behauptet, dass „Dankbarkeit besser als eine Tugend denn als eine Quelle von Pflichten zu verstehen“ und ,die Hauptquelle unserer die Dankbarkeit betreffenden morali- 
schen Wertungen der Handelnde und nicht seine Handlung" sei. Dieser Auffassung folgend könne ,der wohlwollende Ausdruck des Entgegenkommens seitens eines Begünstigenden dem Begünstigten moralische Gründe an die Hand geben, diesem mit gleichem Entgegenkommen zu antworten, wobei [...] diese moralischen Gründe den Begünstigten allerdings nicht der Pflicht überantworten“ (Wellman 1999). Undankbarkeit gehört Wellman zufolge zur Kategorie dessen, was er ,suberogatorisch“ nennt: zu dem, was schlecht, aber nicht verboten ist. Wir haben allgemein betrachtet zwangsläufig keine Pflicht, all das zu tun, was wir tun sollten. Wellman geht sogar so weit, McConnells Vermittlungsposition abzulehnen, der zufolge Dankbarkeit als eine Tugend emotionalen Empfindens, zugleich aber auch als Quelle von Verpflichtungen verstanden werden kann, sich auf eine bestimmte Weise zu äußern.

Carr hat zutreffend bemerkt, dass Wellman womöglich den Unterschied zwischen „gute moralische Gründe für x haben“ und „moralisch zu x aufgefordert sein“ überzeichnet (Carr 2013). Das Problem besteht hier darin, dass Wellman, indem er die Verbindung zwischen Tugend und Verpflichtung vollständig kappt, vielen gegenwärtigen Gegnern der Tugendethik in die Hände spielt, die beanstanden, die Theorie, zumindest in ihrer gegenwärtigen Form, leide an einem Mangel an Handlungsführung (Kristjánsson 2002, Kap. 2.2). In Antwort darauf hat die Tugendethikerin Rosalind Hursthouse betont, wie eine jede Tugend in der Tat eine Anweisung oder Vorschrift generiert (sei dankbar, handele mutig usw.) und jedes Laster ein Verbot (sei nicht undankbar, handele nicht grausam etc.) (Hursthouse 1996). Ferner sollte man sich daran erinnern, dass Aristoteles, der Vater der Tugendethik, eingestanden hat, dass den „Festspielen von Olympia“ gleich, bei denen ,nicht die den Siegeskranz erringen, die am schönsten und stärksten aussehen, sondern die Kämpfer - denn aus ihren Reihen treten die Sieger -“, so auch ,zu den Siegespreisen des Lebens nur die Menschen [gelangen], die richtig handeln" (Aristoteles, NE 1099a). Ungeachtet dieser Überlegungen finden wir unter Moralphilosophen so unterschiedliche Auffassungen zur Natur der Dankbarkeit, dass nicht allein deren ethische Wertung auf dem Spiel steht, sondern vielmehr das Wesen von Dankbarkeit als eines Moralbegriffs überhaupt.

\section{Warum und auf welche Weise ist Dankbarkeit wertvoll?}

Obwohl sich Psychologen kaum an der im vorherigen Abschnitt beschriebenen Debatte beteiligt haben, sind ihre Stimmen in jener nicht zu überhören, die sich mit den relativen moralischen Vorzügen von Dankbarkeit beschäftigt. Wir möchten jedoch mit einem kurzen Verweis auf historische Quellen beginnen. Historisch gesehen hat die Rede von Dankbarkeit aus irgendeinem Grund den Gebrauch von Superlativen hervorgerufen.

Ciceros Darstellung von Dankbarkeit, ,nicht nur die größte aller Tugenden, sondern auch die Mutter von allen übrigen" zu sein, wird oft zitiert. Eine bekannte zeitgenössische Quelle nennt Dankbarkeit ,die leidenschaftlichste Kraft der Umgestaltung im Kosmos“. Im Gegenzug war für Hume Undankbarkeit das ,scheußlichste und unnatürlichste“ von ,allen Verbrechen, die menschliche Geschöpfe zu begehen fähig sind“, während sie für Kant zum „Wesen der Widerwärtigkeit und Bosheit“" 
zählte. Shakespeare schrieb: „du Winterwind/ du bist nicht falsch gesinnt,/ wie Menschenundank ist“. „Undankbarkeit“" war für ihn ,,abscheulicher“ als „Meeresungeheuer" (vgl. für die Zitate McCullough und Tsang 2004, S. 123; Shelton 2004; Emmons 2004a, S. 7; Emmons 2012; McConnell 1993, S. 3). Trotz dieser scheinbaren Übereinstimmung unter den „Weisen“, scheint Bedeutung und Stellenwert von Dankbarkeit unter den „Vielen“ zu weiten Teilen von den zugrunde liegenden Wertbindungen der Menschen abzuhängen (Ceaser 2011). Um Dankbarkeit erfahren zu können, muss der Einzelne in der Lage sein, seinen Status als Empfänger zu akzeptieren. Einzelne, die es schwierig oder erniedrigend finden, von anderen etwas $\mathrm{zu}$ erhalten oder auf sie angewiesen $\mathrm{zu}$ sein, werden eine weniger positive Auffassung von Dankbarkeit besitzen als jene, die bereit sind, auf andere zu setzen (Roberts 2004 und 2007). Sich in einer Position der Abhängigkeit zu befinden, hat für praktizierende Christen eine andere Wertigkeit als für Anhänger von Aristoteles. In welchem Maße Dankbarkeit geschätzt wird, scheint somit durch die Urteile über den relativen Wert von Autonomie und Abhängigkeit bedingt zu sein. Während Menschen, die in starkem Maße zur Autonomie tendieren, eine eher negative Einstellung zur Dankbarkeit zeigen mögen, müssen jene, die gegenseitige Abhängigkeit wirklich schätzen, sicherstellen, dass ihre Zuversicht in andere eher einem wechselseitigen Vertrauen gleicht als einer ungesunden Abhängigkeit von diesen (Shelton 2004).

In einer mittlerweile klassischen Abhandlung zeitgenössischer Psychologie haben McCullough und Mitarbeiter den moralischen Wert von Dankbarkeit unter dem Aspekt von drei Funktionen untersucht: als (1) moralisches Barometer, (2) moralischen Anreiz und (3) moralischen Verstärker (McCullough et al. 2001). Das erste Element, das moralische Barometer, beinhaltet eine Wandlung des moralisch offensichtlichen Affekts des Begünstigten, die auf eine Begünstigung durch andere zurückzuführen ist. Als ein Anreiz drängt uns Dankbarkeit dazu, zukünftig zum Wohl unseres Begünstigenden (oder Dritter) beizutragen. Als Verstärker führt Dankbarkeit dazu, dass Begünstigende mit größerer Wahrscheinlichkeit zu späteren Momenten und in anderen kommunalen Zusammenhängen Akte guten Willens wiederholen. Mittels dieser drei Moralfunktionen generiert Dankbarkeit eine Folge guter Ereignisse [a virtuous circle of events], wobei eine gute Handlung eine andere erzeugt. McCullough und Mitarbeiter haben diese Funktionstheorie nicht nur als ein analytisches Werkzeug vorgestellt, sie haben auch eine große Anzahl empirischer Untersuchungen zur Dankbarkeit geprüft und gezeigt, wie diese die Theorie bestätigen.

Die weitere Forschung hat die Funktionstheorie mit empirischen Belegen gestützt. So ist die Verbindung zwischen Dankbarkeit und moralischem (oder prosozialem) Verhalten in der jüngsten Forschung weiter untersucht worden. Grant und Gino haben analysiert, ob die Beziehung auf ,agentischen“ oder „kommunalen“ Mechanismen beruht, wobei zu agentischen Mechanismen Ausdrücke prosozialen Verhaltens gehören, die durch eine Zunahme von Selbsteffektivität (das Gefühl, dass man zu helfen fähig ist) bedingt sind, und kommunale Mechanismen aufgrund einer Zunahme sozialen Werts (das Gefühl, dass die Gunst akzeptiert und geschätzt wird) zu prosozialem Verhalten führen (Grant, Gino 2010). Auf geschickte Weise haben sie untersucht, ob der Dank für eine Gunst die Selbsteffektivität des Begüns- 
tigenden erhöhen und es wahrscheinlicher machen würde, dass er sich prosozial betätigt, und gleichermaßen, ob der Dank den sozialen Wert des Begünstigenden erhöht und dadurch dessen prosoziales Verhalten stärkt. Sie haben belegen können, dass Dankbarkeit sowohl die Selbsteffektivität als auch den sozialen Wert erhöht und der soziale Wert prosoziales Verhalten vermittelt. Dieser Befund stützt die Annahme von Dankbarkeit als eines moralischen Verstärkers und verweist darauf, dass bei Episoden von Dankbarkeit irgendeine Art kommunaler Mechanismus am Werk ist, der Menschen zusammenbringt und zu weiteren Ereignissen von Dankbarkeit führen kann. Allerdings ist einzugestehen, dass die Literatur der empirischen Psychologie nicht wirklich auf die Frage eingeht, wie die Termini „moralisch“ und ,prosozial“ zu verstehen sind. So bezieht man sich üblicherweise auf Dankbarkeit als ,,moralischen Affekt“ oder darauf, dass Dankbarkeit „,moralisches“ oder ,,achtbares Verhalten“ anregt, ohne dass klar angegeben würde, was den moralischen Affekt oder das moralische Verhalten ausmacht (Naito et al. 2010).

Aus der Perspektive der Tugendethik, die im vorhergehenden Abschnitt erläutert wurde, scheint die Funktionstheorie moralischen Werts vielleicht zu instrumentalistisch $\mathrm{zu}$ sein, hat sie doch über ihre äußeren Vorzüge hinaus zum Eigenwert von Dankbarkeit wenig zu sagen. Fitzgerald rechtfertigt den moralischen Wert von Dankbarkeit in Bezug auf ihre Nützlichkeit (1) bei der Vermeidung von Leid und Belastungen, (2) bei der Beförderung oder dem Erhalt persönlicher oder kommunaler Beziehungen und (3) als Hilfeleistung bei der Entwicklung (anderer) Tugenden (Fitzgerald 1998). Allerdings scheinen diese Gründe kaum weniger instrumentalistisch als jene zu sein, die McCullough und Mitarbeiter anbieten (McCullough et al. 2001). Roberts legt die Aufmerksamkeit auf die Rolle, die Dankbarkeit bei der Milderung der Neigung zu schädigenden und/oder boshaften Emotionen, wie etwa Missgunst, Neid und Kummer, zukommt (Roberts 2004, S. 66-77). Der Stichhaltigkeit seiner Argumente zum Trotz hat dies - von der Tugendethik und der positiven Psychologie her gesehen - etwas untypisch Obskures, wenn nämlich ausschließlich die Wirkungen von Dankbarkeit betont werden, die Unglücklichsein mindern, im Unterschied zu jenen, die Glücklichsein konstituieren. Roberts nachträgliche Darstellung von Dankbarkeit als einer Spiegelung von Großzügigkeit - eine seitenverkehrte Verdoppelung der großzügigen Antwort - weist vielleicht in die richtige Richtung, allerdings noch nicht in zufriedenstellender Weise (Roberts 2007).

Im Ganzen gesehen leidet der Moraldiskurs zur Dankbarkeit sowohl in philosophischen als auch in psychologischen Diskussionskreisen immer noch daran, dass die Unterscheidung zwischen einer instrumentalistischen und intrinsischen Rechtfertigung der fraglichen emotionalen Tugend unklar ist. Während eindeutige Belege konzeptioneller wie empirischer Art für den moralischen Wert von Dankbarkeit wohl vorgelegt worden sind, konnte die Tugend nicht als „,die Mutter aller Tugenden“ erwiesen werden. Im Vergleich mit den Tugenden, die Aristoteles für die bedeutendsten hielt, wie etwa Gerechtigkeit, Großzügigkeit und Hochsinnigkeit, scheint Dankbarkeit immer noch eher eine Magd oder ein Enkel zu sein als eine Mutter. Ferner ist, wie Shelton bemerkt hat, die Erfahrung von Dankbarkeit kein Königsweg zur allseitigen moralischen Tugend. Selbst Hitler wird wohl Gefühle echter Dankbarkeit erfahren haben (Shelton 2004, S. 267). Zweifellos besteht aber, wie in Abschnitt 2 erwähnt, ein bedeutsamer Unterschied zwischen Episoden von Dankbar- 
keit einerseits und Dankbarkeit als einer moralischen Tugend andererseits. Während ein jeder Dankbarkeit als gelegentliche Episode erfahren haben mag, muss man, damit Dankbarkeit als eine Tugend funktioniert, dafür veranlagt sein, Dankbarkeit oft und auf die richtige Weise zu erfahren - man muss Dankbarkeit dann fühlen, wenn dies angemessen ist, man muss sie in angemessener Weise fühlen und von überschwänglicher Dankbarkeit dort Abstand nehmen, wo die Situation dies legitimerweise nicht erlaubt. Darüber hinaus muss man dankbar für die richtigen Sachen sein. Dankbarkeit gegenüber einer kriminellen Vereinigung, die das eigene Drogengeschäft am Laufen hält, erscheint nicht besonders moralisch (ungeachtet dessen, wie stark und oft diese Dankbarkeit erfahren wird).

(...)

\section{Schluss}

In den letzten Jahren ist eine Vielzahl philosophischer Analysen und empirischer Untersuchungen zur Dankbarkeit durchgeführt worden. Während die gegenwärtige Forschung sich überwiegend mit den psychologischen Erträgen von Dankbarkeit oder Dankbarkeits-Interventionen beschäftigt, sind wir zu diesem Diskurs bewusst auf Abstand gegangen und haben uns den zugrundeliegenden begrifflichen Fragen zugewandt. Wir stimmen Lambert, Graham und Fincham zu, dass es (zwischen begrifflichen und operationalen Definitionen) „ernsthafte Meinungsverschiedenheiten“ unter Gelehrten darüber gibt, wie Dankbarkeit zu bestimmen wäre, und dass dieser „Mangel an Bestimmtheit der Theorieentwicklung schadet“" (Lambert et al. 2009, S. 1194 und 1205). Wir haben in dieser Abhandlung die Wurzel dieser Begriffsverwirrung in der mangelnden Aufmerksamkeit gegenüber begrifflichen Fragen in der psychologischen Literatur ermittelt sowie darin, dass Philosophen die von ihnen bevorzugten Darstellungen von Dankbarkeit oktroyieren, ohne sich wirklich dafür zu interessieren, wie der Begriff tatsächlich alltagssprachlich Verwendung findet. Ein häufiges Versehen, das den psychologischen Diskurs nicht weniger stark gefährdet als den philosophischen, besteht darin, die vorrangige Frage, welcher Begriffstyp untersucht wird, nicht zu thematisieren. Handelt es sich um den Sammelbegriff von Dankbarkeit, dem ein gemeinsamer Begriffskern fehlt, oder handelt es sich um einen der vielen Unterbegriffe von Dankbarkeit, die sich in den verschiedenen Publikationen weitverbreitet finden? Die Arbeit mit dem Sammelbegriff würde andere Begriffsmethoden (zum Beispiel Prototypenanalysen) erfordern als jene Art der Begriffsanalyse, der Philosophen typischerweise Unterbegriffe mit einem gemeinsamen Begriffskern unterziehen. (...)

Von entscheidender Bedeutung ist, dass Psychologen, statt emsig wissenschaftliche Abhandlungen über die Effekte von Dankbarkeit zu publizieren, mehr Zeit den zentralen Fragen der Begrifflichkeit widmen. Der aufstrebende Bereich der Dankbarkeitsforschung ist ein spannendes Arbeitsfeld. Allerdings ist die zu leistende Vorarbeit bei weitem noch nicht getan.

Danksagung Diese Veröffentlichung wurde durch Fördermittel der John Templeton Foundation ermöglicht. Die in dieser Abhandlung zum Ausdruck gebrachten Auffassungen sind die der Autoren und spiegeln nicht unbedingt jene der John Templeton Foundation wider. 
Funding Open access funding provided by University of Northampton.

Open Access Dieser Artikel wird unter der Creative Commons Namensnennung 4.0 International Lizenz veröffentlicht, welche die Nutzung, Vervielfältigung, Bearbeitung, Verbreitung und Wiedergabe in jeglichem Medium und Format erlaubt, sofern Sie den/die ursprünglichen Autor(en) und die Quelle ordnungsgemäß nennen, einen Link zur Creative Commons Lizenz beifügen und angeben, ob Änderungen vorgenommen wurden.

Die in diesem Artikel enthaltenen Bilder und sonstiges Drittmaterial unterliegen ebenfalls der genannten Creative Commons Lizenz, sofern sich aus der Abbildungslegende nichts anderes ergibt. Sofern das betreffende Material nicht unter der genannten Creative Commons Lizenz steht und die betreffende Handlung nicht nach gesetzlichen Vorschriften erlaubt ist, ist für die oben aufgeführten Weiterverwendungen des Materials die Einwilligung des jeweiligen Rechteinhabers einzuholen.

Weitere Details zur Lizenz entnehmen Sie bitte der Lizenzinformation auf http://creativecommons.org/ licenses/by/4.0/deed.de.

\section{Literatur}

Adler, Mitchel/Fagley, Nancy. 2005. Appreciation: Individual Differences in Finding Value and Meaning as a Unique Predictor of Subjective Well-Being. Journal of Personality 73 (1), 79-114.

Algoe, Sarah/Haidt, Jonathan/Gable, Shelly. 2008. Beyond Reciprocity: Gratitude and Relationships in Everyday Life. Emotion 8 (3), 425-429.

Allport, G. 1937. Personality: A Psychological Interpretation. New York: Holt.

Aristoteles. Nikomachische Ethik. Übers. v. Franz Dirlmeier. Werke in deutscher Übersetzung, Bd. 6. Berlin: Akademie Verlag 1999.

Aristoteles. Rhetorik. Übers. v. Christof Rapp. Werke in deutscher Übersetzung, Bd. 4. Berlin: Akademie Verlag 2002.

Bartlett, Monica/DeSteno, David. 2006. Gratitude and Prosocial Behavior. Psychological Science 17 (4), 319-325.

Berger, Fred. 1975. Gratitude. Ethics 85 (4), 298-309.

Bertocci, P.A./Millard, R.M. 1963. Personality and the Good: Psychological and Ethical Perspectives. New York: David McKay.

Card, Claudia. 1988. Gratitude and Obligation. American Philosophical Quarterly 25 (2), 115-127.

Carr, David. 2013. Varieties of Gratitude. The Journal of Value Inquiry 47, 17-28.

Ceaser, J.W. 2011. No Thanks to Gratitude. Policy Review 170. Abgerufen am 27.11. 2012. http://www. hoover.org/publications/policy-review/article/100981

Curzer, H.J. 2012. Aristotle and the Virtues. Oxford: Oxford University Press.

Emmons, Robert. 2004a. The Psychology of Gratitude: An Introduction. In R. A. Emmons, M. E. McCullough (Eds.), The Psychology of Gratitude, 3-16. Oxford: Oxford University Press.

Emmons, Robert. 2004b. Gratitude. In M. E. P. Seligman, C. Peterson (Eds.), The VIA Taxonomy of Human Strengths and Virtues, 553-568. Oxford: Oxford University Press.

Emmons, Robert. 2007. Thanks! How Practicing Gratitude Can Make You Happier. New York: Mariner Books.

Emmons, Robert/Crumpler, Cheryl. 2000. Gratitude as a Human Strength: Appraising the Evidence. Journal of Social and Clinical Psychology 19 (1), 56-69.

Emmons, Robert/McCullough, M.E. 2003. Counting Blessings Versus Burdens: An Experimental Investigation of Gratitude and Subjective Well-Being in Daily Life. Journal of Personality and Social Psychology 84 (2), 377-389.

Emmons, Robert/Shelton, Charles. 2002. Gratitude and the Science of Positive Psychology. In C.R. Snyder, S.J. Lopez (Eds.), Handbook of Positive Psychology, 459-471. Oxford: Oxford University Press.

Emmons, Robert. 2012. Queen of the Virtues? Gratitude as a Human Strength. Reflective Practice: Formation and Supervision in Ministry 32. Abgerufen am 27.11.2012. http://journals.sfu.ca/rpfs/index.php/ rpfs/article/viewFile/59/58

Fagley, Nancy. 2012. Appreciation Uniquely Predicts Life Satisfaction Above Demographics: The Big 5 Personality Factors, and Gratitude. Personality and Individual Differences 53 (1), 59-63.

Fitzgerald, Patrick. 1998. Gratitude and Justice. Ethics 109 (3), 119-153. 
Fredrickson, Barbara. 2004. Gratitude, Like Other Positive Emotions, Broadens and Builds. In Robert Emmons, M.E. McCullough (Eds.), The Psychology of Gratitude, 145-166. Oxford: Oxford University Press.

Fredrickson, Barbara. 2009. Positivity: Groundbreaking Research Reveals How to Embrace the Hidden Strength of Positive Emotions, Overcome Negativity, and Thrive. New York: Crown.

Froh, Jeffrey/Emmons, Robert/Card, Noel/Bono, Giacomo/Wilson, Jennifer. 2011.Gratitude and the Reduced Costs of Materialism in Adolescents. Journal of Happiness Studies 12 (2), 289-302

Gordon, Anne/Musher-Eizenman, Dara/Holub, Shayla/Dalrymple, John. 2004. What Are Children Thankful for? An Archival Analysis of Gratitude Before and After the Attacks of September 11 ${ }^{\text {th }}$. Journal of Applied Development Psychology 25 (5), 541-553.

Grant, Adam/Gino, Francesca. 2010. A Little Thanks Goes a Long Way: Explaining Why Gratitude Expressions Motivate Prosocial Behavior. Journal of Personality and Social Psychology 98 (6), 946-955.

Greenberg, Martin/Westcott, David. 1983. Indebtedness as a Mediator of Reactions to Aid. In J. D. Fisher, A. Nadler, B. M. de Paulo (Eds.), New Directions in Helping. Bd. 1, 85-106. New York: Academic Press, 1983.

Gregg, Aiden/Hart, Claire/Sedikides, Constantine/Kumashiro, Madoka. 2008. Everyday Conceptions of Modesty: A Prototype Analysis. Personality and Social Psychology Bulletin 34 (7), 978-992.

Gulliford, Liz. 2013. The Head and the Heart of the Matter in Hope and Forgiveness. In F. N. Watts, G. Dumbreck (Eds.), Head and Heart: Perspectives from Religion and Psychology, 273-312. West Conshohocken, PA: Templeton Press.

Harpham, E.J. 2004. Gratitude in the History of Ideas. In R. A. Emmons, M. E. McCullough (Eds.), The Psychology of Gratitude, 19-36. Oxford: Oxford University Press.

Heider, F. 1958. The Psychology of Interpersonal Relations. New York: Wiley.

Hibbs, Thomas. 2009. Creation, Gratitude and Virtue. Journal of Law, Philosophy and Culture 3 (1), 101-114.

Hursthouse, Rosalind. 1996. Normative Virtue Ethics. In R. Crisp (Ed.), How Should One Live? Essays on the Virtues, 19-36. Oxford: Clarendon Press.

Kristjánsson, Kristján. 1996. Social Freedom: The Responsibility View. Cambridge: Cambridge University Press.

Kristjánsson, Kristján. 2002. Justifying Emotions: Pride and Jealousy. London: Routledge.

Kristjánsson, Kristján. 2007. Aristotle, Emotions, and Education. Aldershot: Ashgate.

Kristjánsson, Kristján. 2013. Virtues and Vices in Positive Psychology: A Philosophical Critique. Cambridge: Cambridge University Press.

Kristjánsson, Kristján. 2015. An Aristotelian Virtue of Gratitude. Topoi 34, 499-511.

Lambert, Nathaniel/Graham, Steven/Fincham, Frank. 2009. A Prototype Analysis of Gratitude: Varieties of Gratitude Experiences. Personality and Social Psychology Bulletin 35 (9), 1193-1207.

McAleer, Sean. 2012. Propositional Gratitude. American Philosophical Quarterly 49, 55-66.

McConnell, Terrance. 1993. Gratitude. Philadelphia, PA: Temple University Press.

McCullough, Michael/Tsang, Jo-Ann. 2004. Parent of the Virtues? The Prosocial Contours of Gratitude. In R. A. Emmons, M. E. McCullough (Eds.), The Psychology of Gratitude. Oxford: Oxford University Press.

McCullough, Michael/Emmons, Robert/Tsang, Jo-Ann. 2002. The Grateful Disposition: A Conceptual and Empirical Topography. Journal of Personality and Social Psychology 82 (1), 112-127.

McCullough, Michael/Kilpatrick, Shelley/Emmons, Robert/Larson, David. 2001. Is Gratitude a Moral Affect? Psychological Bulletin 127 (2), 249-266.

McCullough, Michael/Kimeldorf, Marcia/Cohen, Adam. 2008. An Adaptation for Altruism the Social Causes, Social Effects, and Social Evolution of Gratitude. Current Directions in Psychological Science 17 (4), 281-285.

McCullough, Michael/Tsang, Jo-Ann/Emmons, Robert. 2004. Gratitude in Intermediate Affective Terrain: Links of Grateful Moods to Individual Differences and Daily Emotional Experience. Journal of Personality and Social Psychology 86 (2), 295-309.

Naito, Takashi/Matsuda, Tomoko/Intasuwan, Pachongchit/Chuawanlee, Wiladlak/ Thanachanan, Supaporn/Ounthitiwat, Juran/ Fukushima, Meiko. 2010. Gratitude for, and Regret Toward, Nature: Relationships to Proenvironmental Intent of University Students from Japan. Social Behavior and Personality 38 (7), 993-1008.

Nisters, Thomas. 2012. Utrum gratitudo sit virtus moralis vel passio animae or: Gratitude - An Aristotelian Virtue or an Emotion? In R. Edmondson, K. Hülser (Eds.), Politics of Practical Reasoning, 65-78. Lanham: Lexington Books. 
Oakley, J. 1992. Morality and the Emotions. London: Routledge.

Peterson, Christopher/Martin Seligman. 2004. Character Strengths and Virtues: A Handbook and Classification. Oxford: Oxford University Press.

Roberts, Robert C. 1988. What an Emotion is: A Sketch. Philosophical Review 97 (1), 183-209.

Roberts, Robert C. 2003. Emotions: An Essay in Aid of Moral Psychology. Cambridge: Cambridge University Press.

Roberts, Robert C. 2004. The Blessings of Gratitude: A Conceptual Analysis. In: R. Emmons, M. E. McCullough (Eds.), The Psychology of Gratitude, 58-78. Oxford: Oxford University Press.

Roberts, Robert C. 2007. Gratitude and Generosity. Unveröffentlichtes Typoskript. Vorgestellt auf einer Konferenz über Emotion, Selbst und Identität. Aarhus University, Dänemark.

Roberts, Robert C. 2012. Narrative Ethics. Philosophy Compass 7 (3), 174-182.

Roberts, Robert C. 2013. Generosity and Gratitude in Bleak House, paper delivered at a Jubilee Centre Seminar, University of Birmingham, February 6th 2013.

Seligman, Martin/Steen, Tracy/Park, Nansook/Peterson, Christopher. 2005. Positive Psychology Progress: Empirical Validation of Interventions. American Psychologist 60 (5), 410-421.

Shelton, Charles. 2004. Gratitude: Considerations from a Moral Perspective. In R. A. Emmons, M. E. McCullough (Eds.), The Psychology of Gratitude, 259-281. Oxford: Oxford University Press.

Simmons, A.J. 1979. Moral Principles and Political Obligations. Princeton: Princeton University Press.

Steindl-Rast, David. 2004. Gratitude as Thankfulness and as Gratefulness. In R. A. Emmons, M. E. McCullough, The Psychology of Gratitude. Oxford: Oxford University Press.

Tesser, Abraham/Gatewood, Robert/Driver, Michael. 1968. Some Determinants of Gratitude. Journal of Personality and Social Psychology 9 (3), 233-236.

Tillich, Paul. 1964. Das Ewige im Jetzt. Religiöse Reden. Stuttgart: Evangelisches Verlagswerk.

Tsang, Jo-Ann. 2006. The Effects of Helper Intention on Gratitude and Indebtedness. Motivation and Emotion 30 (3), 198-204.

Tsang, Jo-Ann/Schulwitz, Ashleigh/Carlisle, Robert. 2012. An Experimental Test of the Relationship Between Religion and Gratitude. Psychology of Religion and Spirituality 4 (1), 40-55.

Walker, A.D.M. 1980-81. Gratefulness and Gratitude. Proceedings of the Aristotelian Society 81 (1), 39-55.

Watkins, Philip/Scheer, Jason/Ovnicek, Melinda/Kolts, Russell. 2006. The Debt of Gratitude: Dissociating Gratitude and Indebtedness. Cognition \& Emotion 20 (2), 217-241.

Watkins, Philip/Woodward, Kathrane/Stone, Tamara Stone/Kolts, Russell. 2003. Gratitude and Happiness: Development of a Measure of Gratitude und Relationships with Subjective Well-Being. Social Behavior and Personality 31 (5), 431-451.

Wellman, Christopher. 1999. Gratitude as a Virtue. Pacific Philosophical Quarterly 80 (3), 284-300.

Wittgenstein, Ludwig. 1984. Philosophische Untersuchungen. Werkausgabe Band 1, 225-580. Frankfurt/ Main: Suhrkamp.

Wood, Alex/Joseph, Stephen/Linley, P. Alex. 2007. Coping Style as a Psychological Resource of Grateful People. Journal of Social and Clinical Psychology 26 (9), 1076-1093.

Wood, Alex/Maltby, John/Stewart, Neil/Joseph, Stephen. 2008a. Conceptualizing Gratitude and Appreciation as a Unitary Personality Trait. Personality and Individual Differences 44 (7), 621-632.

Wood, Alex/Joseph, Stephen/Maltby, John. 2008b. Gratitude Uniquely Predicts Satisfaction with Life: Incremental Validity Above the Domains and Facets of the Five Factor Model. Personality and Individual Differences 45 (1), 49-54.

Wood, Alex/Joseph, Stephen/Lloyd, Joanna/Atkins, Samuel. 2009. Gratitude Influences Sleep Through the Mechanism of Pre-sleep Cognitions. Journal of Psychosomatic Research 66 (1), 43-48.

Wood, Alex/Froh, Jeffrey/Geraghty, Adam. 2010. Gratitude and Well-Being: A Review and Theoretical Integration. Clinical Psychology Review 30 (7), 890-905. 